\title{
Integrated transcriptomic analysis of human tuberculosis granulomas and a biomimetic model identifies therapeutic targets
}

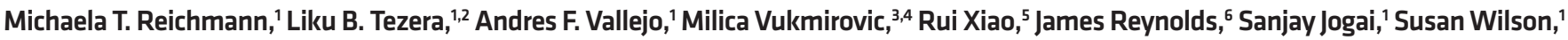 \\ Ben Marshall, ${ }^{1}$ Mark G. Jones, ${ }^{1}$ Alasdair Leslie, ${ }^{2,7}$ Jeanine M. D’Armiento, ${ }^{5}$ Naftali Kaminski, ${ }^{4}$ Marta E. Polak, ${ }^{1,8}$ and Paul Elkington ${ }^{1,8}$ \\ ${ }^{1}$ NIHR Biomedical Research Center, School of Clinical and Experimental Sciences, Faculty of Medicine, University of Southampton, Southampton, United Kingdom. ²Department of Infection and Immunity, \\ University College London, London, United Kingdom. ${ }^{3}$ Firestone Institute for Respiratory Health-Division of Respirology, Department of Medicine, McMaster University, Hamilton, Ontario, Canada. \\ ${ }^{4}$ Department of Medicine, Yale School of Medicine, New Haven, Connecticut, USA. ${ }^{5}$ Columbia University Medical Center, New York, New York, USA. ${ }^{6}$ Ground Upwards Ltd, Winchester, United Kingdom. ${ }^{7}$ Africa \\ Health Research Institute, KwaZulu Natal, South Africa. ${ }^{8}$ Institute for Life Sciences, University of Southampton, Southampton, United Kingdom.
}

Tuberculosis (TB) is a persistent global pandemic, and standard treatment for it has not changed for 30 years. Mycobacterium tuberculosis (Mtb) has undergone prolonged coevolution with humans, and patients can control Mtb even after extensive infection, demonstrating the fine balance between protective and pathological host responses within infected granulomas. We hypothesized that whole transcriptome analysis of human TB granulomas isolated by laser capture microdissection could identify therapeutic targets, and that comparison with a noninfectious granulomatous disease, sarcoidosis, would identify disease-specific pathological mechanisms. Bioinformatic analysis of RNAseq data identified numerous shared pathways between TB and sarcoidosis lymph nodes, and also specific clusters demonstrating TB results from a dysregulated inflammatory immune response. To translate these insights, we compared 3 primary human cell culture models at the whole transcriptome level and demonstrated that the 3D collagen granuloma model most closely reflected human TB disease. We investigated shared signaling pathways with human disease and identified 12 intracellular enzymes as potential therapeutic targets. Sphingosine kinase 1 inhibition controlled Mtb growth, concurrently reducing intracellular pH in infected monocytes and suppressing inflammatory mediator secretion. Immunohistochemical staining confirmed that sphingosine kinase 1 is expressed in human lung TB granulomas, and therefore represents a host therapeutic target to improve TB outcomes.

\section{Introduction}

Globally, tuberculosis (TB) kills 1.4 million people each year, and in 2019 it was the leading cause of mortality due to a single infectious agent (1). TB primarily affects young people of working age, resulting in a disproportionately high socioeconomic burden. Mycobacterium tuberculosis (Mtb) causes TB and has undergone prolonged coevolution with humans, essentially resulting in a symbiotic

\footnotetext{
Authorship note: MEP and PE are co-senior authors.
}

Conflict of interest: NK has served as a consultant to Biogen Idec, Boehringer Ingelheim, Third Rock, Pliant, Samumed, NuMedii, Theravance, LifeMax, Three Lake Partners, Optikira, Astra Zeneca, Veracyte, Augmanity, and CSL Behring, and reports equity in Pliant and grants from Veracyte, Boehringer Ingelheim, and Bristol Myers Squibb. NK has received nonfinancial support from MiRagen and Astra Zeneca. NK owns intellectual property on novel biomarkers and therapeutics in pulmonary fibrosis and ARDS licensed to Biotech: "Biomarkers for assessing idiopathic pulmonary fibrosis" (patent 10961582); "52-gene signature in peripheral blood identifies a genomic profile associated with increased risk of mortality and poor disease outcomes in idiopathic pulmonary fibrosis" (patent 10896740); "Methods of treating or preventing acute respiratory distress syndrome" (publication 20210008020); "Methods of treating or preventing fibrotic lung diseases" (patent 10792265); "Compositions and methods of treating or preventing fibrotic lung diseases" (publication 20200113863). Copyright: This is an open access article published under the terms of the Creative Commons Attribution 4.0 International License.

Submitted: January 28, 2021; Accepted: June 11, 2021; Published: August 2, 2021. Reference information: / Clin Invest. 2021;131(15):e148136. https://doi.org/10.1172/JCl148136. relationship between Mtb and its host (2). Granuloma formation is the hallmark pathological feature of TB, typically consisting of a caseous necrotic core surrounded by macrophages and an outer T cell infiltrate $(3,4)$. Granulomas have traditionally been thought to protect the host, but recent evidence suggests granulomas may also benefit the pathogen (3). Sarcoidosis is another human granulomatous disease, which shares many histological and clinical features with TB, primarily affecting the lungs and lymph nodes (5). The etiology of sarcoidosis remains undetermined, but is likely to involve autoimmune mechanisms (6). We hypothesized that comparative analysis of TB, caused by Mtb infection, and sarcoidosis, a noninfectious granulomatous disease, would identify TB-specific disease mechanisms and novel therapeutic targets.

TB causes morbidity and mortality due to the inflammatory host immune response to the pathogen, resulting in lung matrix destruction and transmission (7). However, in the preantibiotic era, approximately one-third of patients with pulmonary TB spontaneously recovered, indicating the fine balance between protective and pathological host responses (8). Consequently, the concept of host-directed therapy (HDT) for TB has emerged, aiming to improve the clinical outcomes of TB by enhancing an efficacious immune response (9). Clinical trials using repurposed drugs such as metformin, pravastatin, vitamin $\mathrm{D}$, doxycycline, and imatinib have been initiated, with each study emerging from a hypothesis-based approach (10). As Mtb is a pathogen exclusive to humans, 


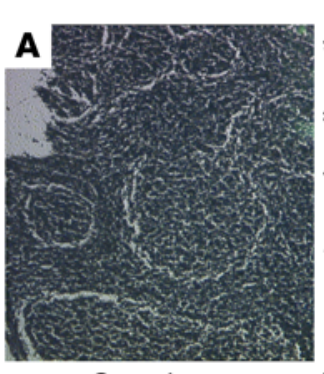

Granuloma

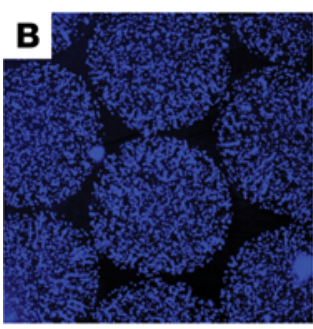

PBMCs cultured

in $3 \mathrm{D}$ microspheres
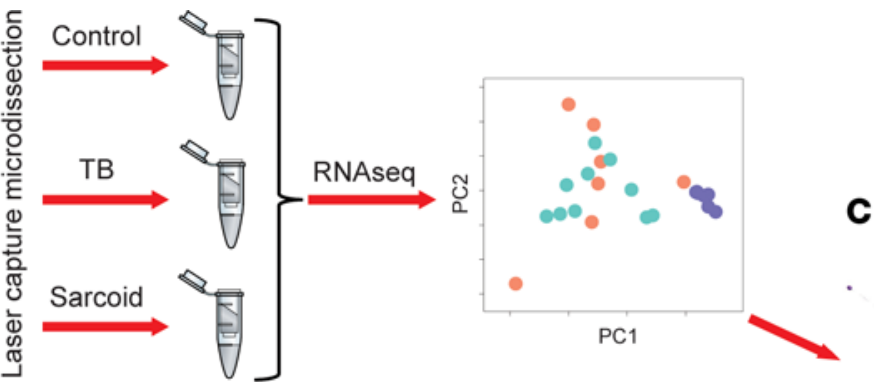

C

Bioinformatic analysis
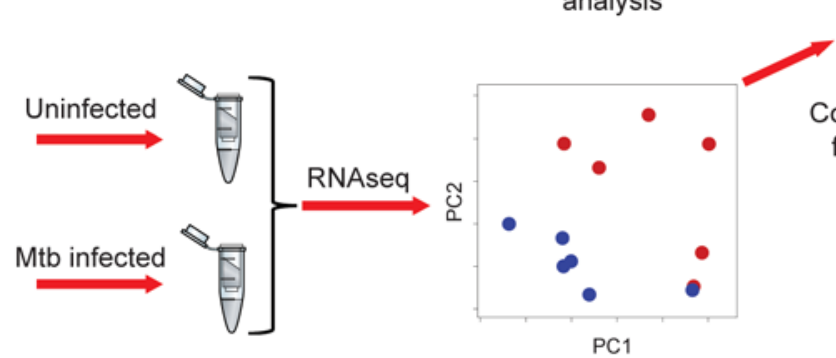

Comp ve analysis or target pathway identification

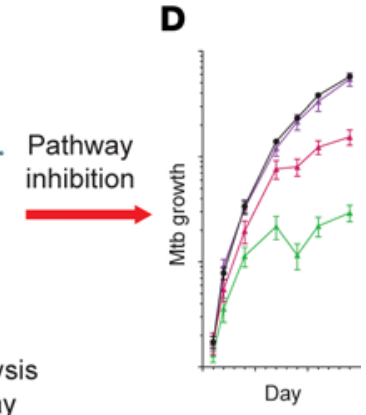

Figure 1. Overall study design. (A) Granulomas from human biopsy specimens were acquired by laser capture microdissection, RNA was extracted and sequenced using the lon Torrent system. (B) Human PBMCs were infected overnight with Mtb and encapsulated in a 3D collagen model, and RNA was extracted at day 4 and sequenced using the Illumina HiSeq system. (C) Bioinformatic analysis was performed to analyze and correlate the clinical specimen and in vitro model data sets to identify key regulatory pathways. (D) Pathways upregulated in patients with TB were inhibited in the 3D model to determine the effect on the host-pathogen interaction.

we analyzed treatment-naive clinical lymph node TB specimens alongside sarcoidosis samples to dissect disease mechanisms, and then studied a biomimetic granuloma model in order to identify novel HDT targets in an unbiased manner.

\section{Results}

Identification and RNAseq analysis of TB, sarcoidosis, and control lymph node biopsies. The overall study design is presented in Figure 1. We searched our biorepository of lymph node samples taken during the clinical diagnostic pathway, with a subsequent diagnosis of tuberculosis, sarcoidosis and normal tissue. We used a screening process to identify specimens with robust diagnosis, no confounding clinical factors, and consistency of biopsy date (Supplemental Figure 1; supplemental material available online with this article; https://doi.org/10.1172/JCI148136DS1). Lymph node biopsies were analyzed from 7 untreated TB patients, 10 untreated sarcoidosis patients, and 7 control samples. Granulomas were acquired using laser capture microdissection until a total area of $8 \mathrm{~mm}^{2}$ per donor was acquired (Supplemental Figure 2), and an equivalent surface area was sampled from control lymph nodes. RNA sequencing (RNAseq) was performed using Ion Semiconductor Sequencing, and the data were explored for variance, normalized, and checked for outliers (Supplemental Figure 3). No outliers were identified after normalization.

Tuberculosis and sarcoidosis granulomas share transcriptional programs. Initial exploratory data analysis by principal component analysis identified a high degree of overlap between TB and sarcoidosis samples, in contrast to clear clustering of control lymph node samples (Figure 2A). Analysis according to other variables including sex, ethnicity, site of lymph node, day of sequencing, or sequencing chip did not identify any confounding effects (Supplemental Figure 4). Hierarchical clustering of the 50 most variable genes also demonstrated clear separation of control samples, but did not separate TB and sarcoidosis samples (Figure 2B and gene list Supplemental Table 1). A similar pattern was observed with analysis of the 1000 most variable genes (Supplemental Figure 5 and Supplemental Table 2).

Analysis identified significant numbers of differentially expressed genes (DEGs) in TB and sarcoidosis relative to control samples. In the TB samples, 748 genes were upregulated, of which 376 were shared with sarcoidosis (Figure 2C and Supplemental Table 3 ), while 111 genes were sarcoidosis unique. Among the genes downregulated, overlap was less marked, with 154 shared, 382 specific to $\mathrm{TB}$, and 168 to sarcoidosis (Figure 2D and Supplemental Table 4). Of the total 1563 DEGs, one-third of them were shared between TB and sarcoidosis. Analysis of fold change and significance estimated as false discovery rate (FDR) less than 0.05 demonstrated $\log _{2}$ changes of up to 11-fold in TB relative to control samples (Figure 2E, gene list Supplemental Table 5), whereas the degree fold change in sarcoidosis was less marked (Figure $2 \mathrm{~F}$ and Supplemental Table 6). Plotting DEGs in TB relative to control samples onto the KEGG TB pathway showed 79 of 139 genes to be upregulated (Supplemental Figure 6).

Direct comparison of $\mathrm{TB}$ and sarcoidosis was performed to identify differences between the 2 granulomatous conditions (Supplemental Table 7). Gene ontology analysis demonstrated that the primary biological processes upregulated in TB relative to sarcoidosis involve cytokine signaling and the inflammatory response, angiogenesis, and extracellular matrix organization 
A

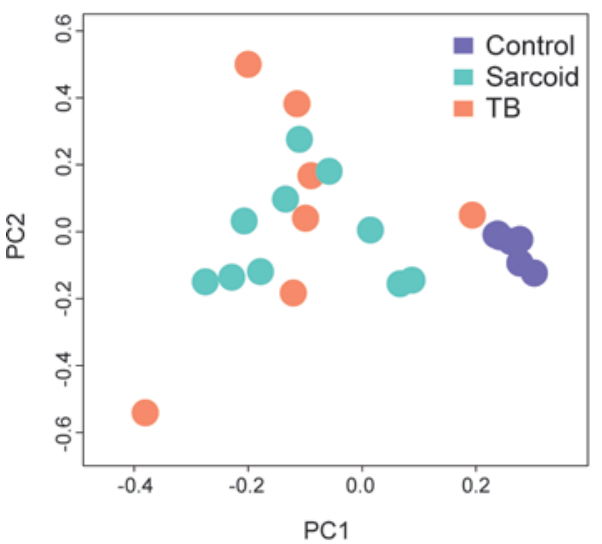

C

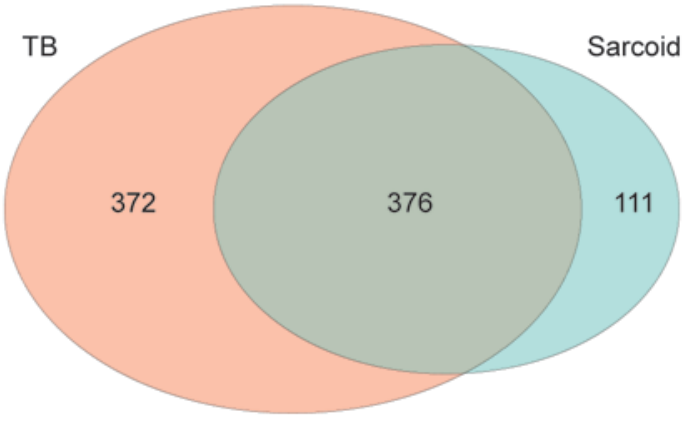

Upregulated

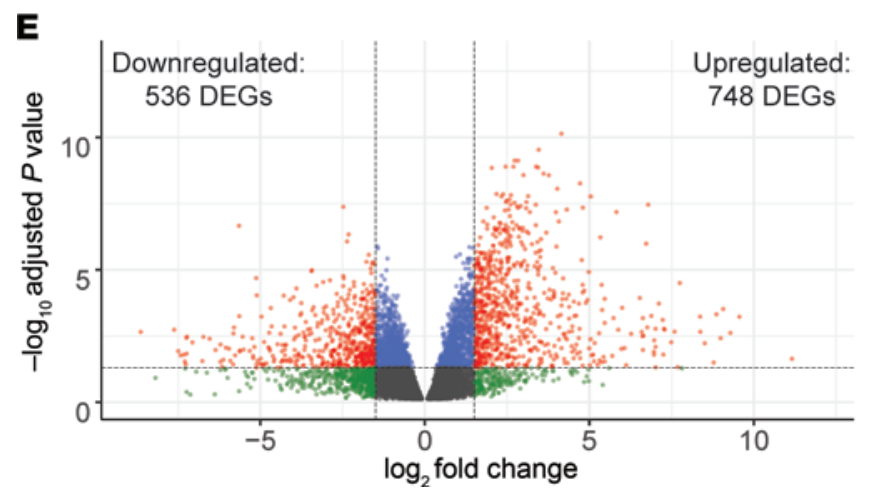

B
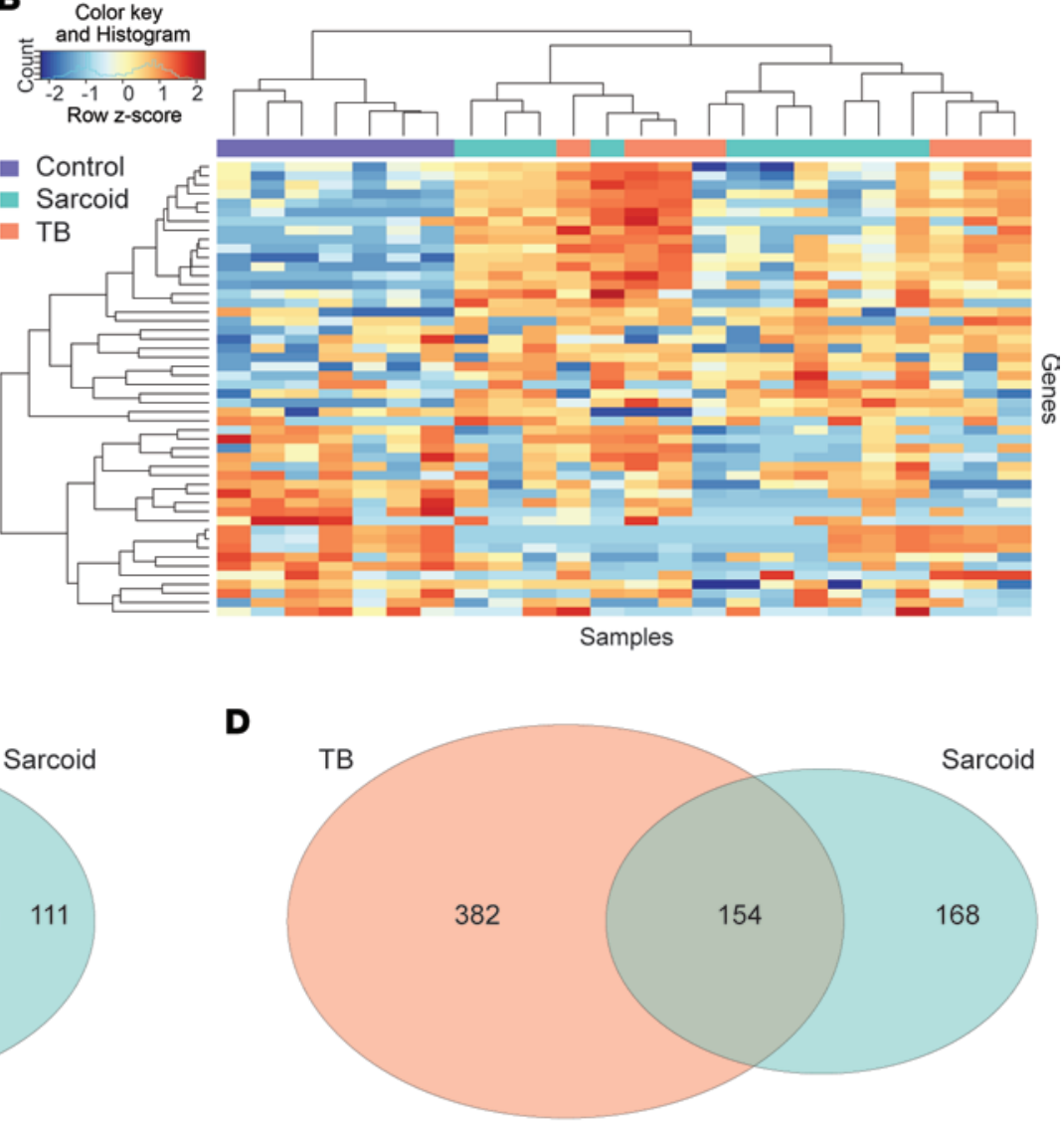

Downregulated

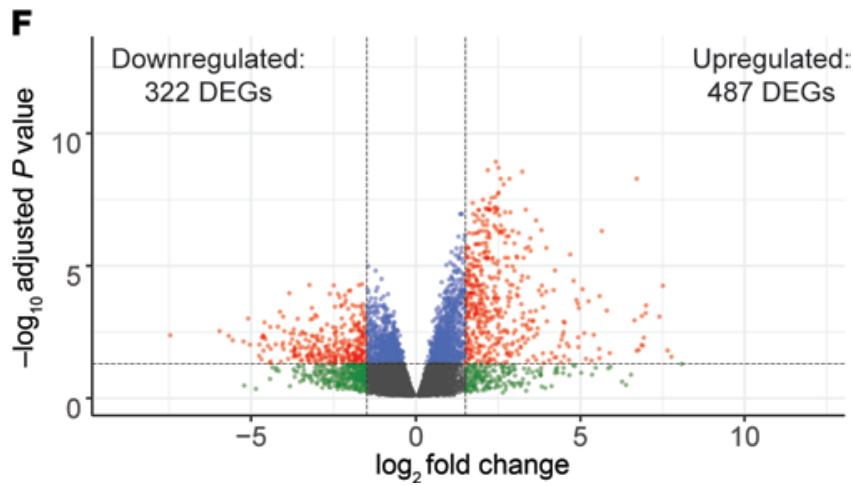

Figure 2. Gene expression in TB and sarcoidosis has significant overlap. (A) PCA plot of whole transcriptome data demonstrates separation of the control group from diseased lymph nodes (purple), while the TB (orange) and sarcoidosis (blue) samples overlap. (B) Hierarchical clustering heat map of top 50 most variable genes using Spearman correlation and complete linkage. Control samples (purple) cluster separately to the TB (orange) and sarcoidosis (blue) samples, which show no differentiation. (C) Venn diagram of the number of upregulated genes in TB (orange) and sarcoidosis (blue) relative to control, confirming numerous shared genes ( $\log _{2}$ fold change $\geq 1.5$, adjusted $P$ value $<0.05$ ). (D) Venn diagram of the number of downregulated genes in TB (orange) and sarcoidosis (blue) relative to control $\left(\log _{2}\right.$ fold change $\geq 1.5$, adjusted $P$ value $<0.05$ ). (E and $\mathbf{F}$ ) Volcano plots of differentially expressed genes in TB $(\mathbf{E})$ and sarcoidosis (F) plotting the $\log _{2}$ fold change on the $x$ axis and adjusted $P$ value on the $y$ axis. Gray: similarly expressed genes; green: genes with absolute log fold change $\geq 1.5$; blue: genes with adjusted $P$ value $<0.05$; red: genes exceeding both thresholds. More genes are upregulated in TB, and to a higher fold change, than in sarcoidosis. Horizontal dashed line denotes adjusted $P$ value of 0.05 , vertical dashed line denotes absolute $\log _{2}$ fold change of 1.5.

(Supplemental Figure 7A). The most significantly upregulated process was the cytokine-mediated signaling pathway, including many genes already implicated in TB pathogenesis, such as IL1B, CCL2, CXCL9, CXCL1O, HIF1A, and MMP1 (11), along with novel genes such as SPHK1. Within this group, the most highly upregulated gene was MMP1 (Matrix Metalloproteinase 1; Supplemental Figure 7B). Gene set enrichment analysis identified the lytic vacuole membrane (Supplemental Figure 8A) and KEGG lysosome pathway (Supplemental Figure 8B) as the only components downregulated in TB relative to sarcoidosis. 
A

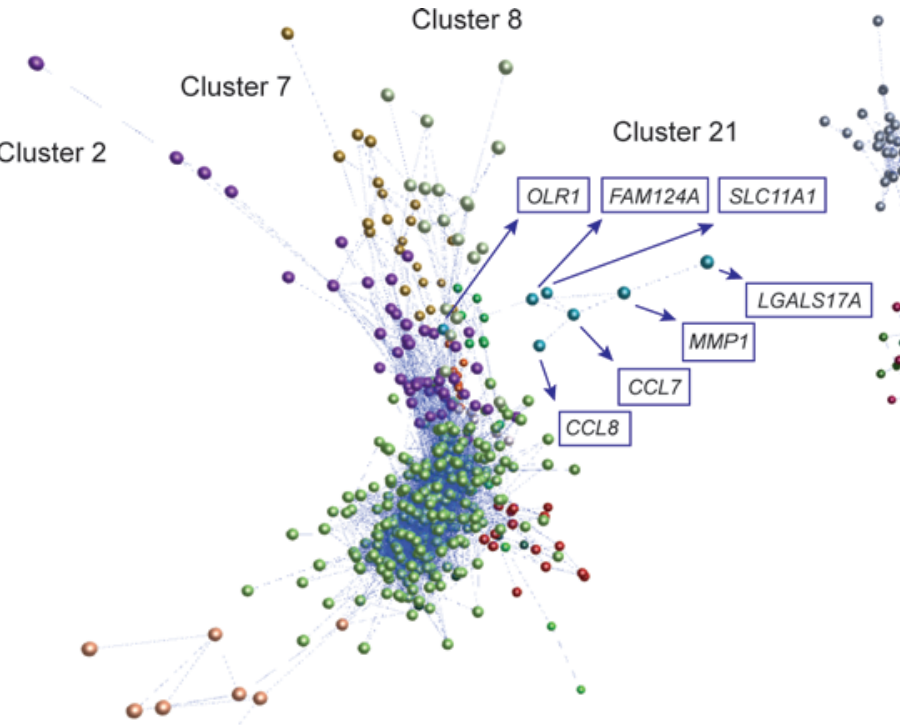

Cluster 14 。
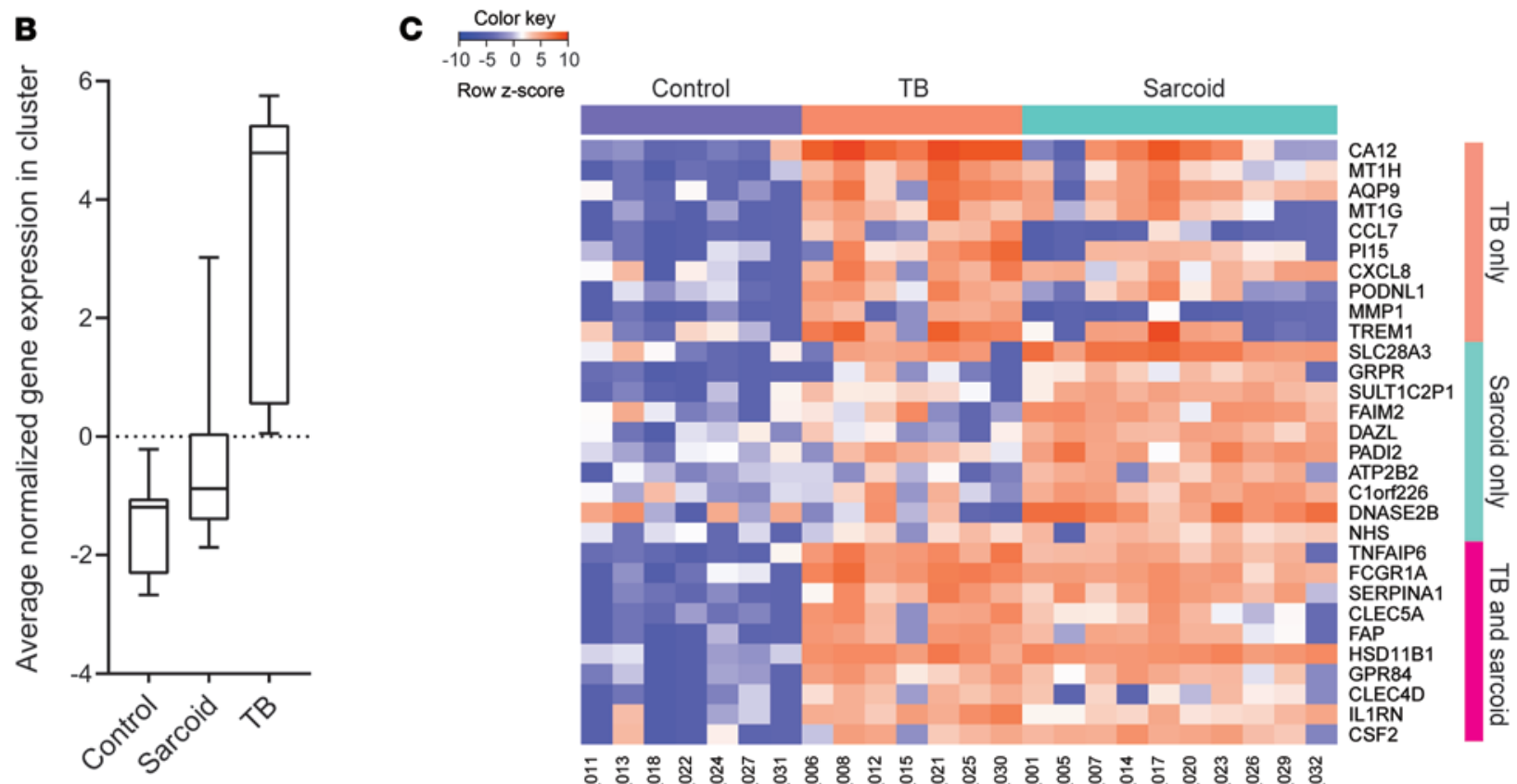

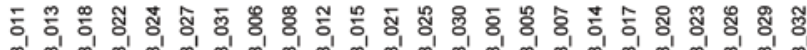

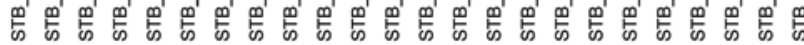

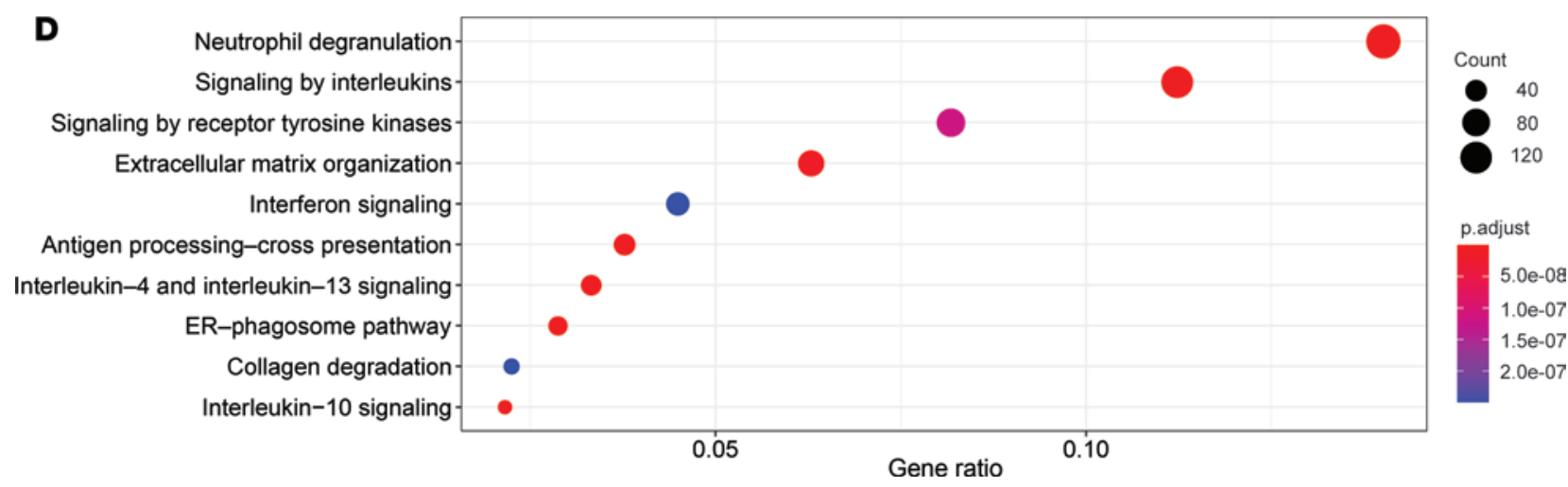


Figure 3. TB and sarcoidosis have disease-specific gene clusters. (A) Correlation analysis performed using Markov Cluster Algorithm (Pearson's $R$ of 0.83 ) with genes of absolute $\log _{2}$ fold change $\geq 1.5$ and adjusted $P$ value $<0.05$. Each node (circle) depicts a transcript/gene, each edge (line) depicts Pearson's correlation value. The left branches display clusters upregulated in TB and sarcoidosis, and the right branches display clusters that are downregulated. Several coregulated clusters are observed. Cluster 21 (blue) is the only cluster specific to TB and comprises the 7 annotated genes. (B) Average (mean) normalized gene expression level in Cluster 21 comparing control $(n=7)$, sarcoidosis $(n=10)$, and TB samples $(n=7)$. Gene expression values after TMM normalization used. Box-and-whisker plot with median values (line). Whiskers represent minimum and maximum values, boxes represent the 25th to 75 th percentiles. (C) Heat map arranged according to top 10 disease-specific upregulated genes based on fold change. Using TB-specific (orange), sarcoidosis-specific (blue), and jointly regulated genes (pink), a clear distinction between control, TB, and sarcoidosis is observed. (D) Gene ontology enrichment (ReactomePA program in R, using genes with adjusted $P$ value $<0.05$ ) showing the top 10 upregulated REACTOME pathways in TB relative to control. Immune processes and extracellular matrix turnover are highly represented. Dot size represents number of expressed genes in the pathway, shade of color represents adjusted $P$ value.

A TB-unique cluster primarily relates to inflammation and the extracellular matrix. Correlation analysis using Markov Cluster Algorithm (Graphia Pearson $r=0.83, \mathrm{MCL}=1.7$ ) of DEGs identified in comparison of the 3 clinical groups (TB relative to control, sarcoidosis relative to control, and TB relative to sarcoidosis samples) (Supplemental Tables 3 and 4) demonstrated multiple clusters of upregulated and downregulated genes (Figure 3A). Of note, Cluster 21 was the only one uniquely upregulated in TB (Figure 3B). Cluster 21 comprised 7 genes including $M M P 1$, plus the divalent transition metal transporter SLC11A1 (solute carrier family 11 member 1, formerly known as NRAMP1), monocyte chemoattractants CCL7 and CCL8 (C-C motif chemokine ligand 7 and 8), OLR1 (oxidized low density lipoprotein receptor 1, formerly known as LOX1), FAM124A (family with sequence similarity 124 member A), and LGALS17A (galectin 14 pseudogene). Gene ontology performed on these 7 genes generated multiple biological processes and pathways with significance after correcting for FDR, implicating a central role for the inflammatory response and extracellular matrix turnover in TB pathogenesis (Supplemental Table 8).

Further analysis of TB-predominant Clusters 2, 7, and 8 identified that the inflammatory response, extracellular matrix, wound healing, and nucleotide metabolism feature significantly on gene ontology analysis (Supplemental Figure 9, A-C and Supplemental Table 9). In contrast, the only sarcoidosis-predominant gene cluster, Cluster 14, comprised 9 genes such as ATP6VOD2 (ATPase H+ transporting VO subunit D2), CHIT1 (Chitinase 1), and FAIM2 (Fas apoptotic inhibitory molecule 2). Analysis of cellular components using gene ontology demonstrated overexpression of the lysosome, but did not generate any significant biological processes or pathways using the same analytical approach (Supplemental Figure 9D and Supplemental Table 9).

Contrasting the expression of the top 10 DEGs most significantly upregulated for each condition, that are specific to TB only, sarcoidosis only, or communal to both according to fold change clearly separated the groups (Figure $3 \mathrm{C}$ and Supplemental Table 10). However, except for $C C L 7$ and $M M P 1$, all genes in the TB only group (orange) were also overexpressed in the sarcoidosis samples (blue). Inspection of individual genes revealed that the TB-specific DEGs are mainly located in the extracellular environment and are often metal binding, while sarcoidosis only DEGs are located intracellularly or at the plasma membrane and are often associated with neural development and protein synthesis (Supplemental Table 10). DEGs shared by both diseases are mainly located extracellularly and relate to the immune response or the extracellular matrix (Supplemental Table 10).

Analyses of the top 10 upregulated REACTOME pathways in TB relative to control samples confirmed immunological pathways and extracellular matrix turnover as predominant processes (Figure 3D). In contrast, downregulated genes in TB primarily relate to chromatin organization and RNA and protein metabolism (Supplemental Figure 10). Gene set enrichment analysis of the top 10 canonical pathways in both TB and sarcoidosis demonstrated the most overexpressed pathways relative to control samples were extracellular matrix-related, both in TB (Supplemental Figure 11A) and sarcoidosis (Supplemental Figure 11B). As this analysis was from lymph node biopsies, we investigated compartment-specific effects relative to published data sets that used RNAseq methodology to allow a consistent analysis pipeline $(12,13)$. Analysis of the lung RNAseq study identified a total of 29 statistically significant upregulated REACTOME pathways in TB (Supplemental Table 11), whereas our microdissection approach of lymph nodes identified 278 pathways (Supplemental Table 12). The lung study was smaller, studying 5 TB samples; included patients already on antibiotic treatment; and did not capture the granuloma microenvironment by microdissection, potentially reducing power and introducing confounders. The top 10 pathways upregulated in the lung were replicated in the lymph node analysis more frequently than the peripheral circulation, while several of the downregulated pathways in the lung were upregulated in blood (Supplemental Figure 12).

The 3D collagen granuloma model most closely replicates gene expression in clinical TB samples. To translate the insights from clinical sample analysis toward new therapeutic targets, we proceeded to perform cell culture studies. In order to determine a model of $\mathrm{TB}$ that can best represent clinical TB, we first compared 3 primary human PBMC cell culture models using the same bioinformatic pipeline as applied to the clinical sample analysis. The 2D model studied was PBMCs infected with Mtb under standard tissue culture conditions, while both 3D models involved overnight infection of PBMCs with Mtb (day 0) and then resuspension in matrix that was encapsulated into 3D microspheres by bioelectrospray methodology. Cells in the 3D alginate model were encapsulated in alginate matrix, whereas the 3D collagen model used alginate-collagen matrix as previously described (14). Within this system, Mtb is phagocytosed by monocytes (15), which then differentiate into $\mathrm{CD}^{+} 8^{+}$macrophages over time (14). The change in gene expression 4 days after Mtb infection relative to uninfected cells was compared with clinical TB samples for upregulated (Supplemental Figure 13A and Supplemental Table 13) and downregulated DEGs (Supplemental Figure 13B and Supplemental Table 14). Overall, the 2D model displayed the highest number of DEGs with 944 genes, though only one-tenth of these overlapped with clinical TB samples, with 79 upregulated and 21 downregulated genes. The 3D alginate model was the most inert model, with 100 DEGs observed, of which 49 upregulated and 1 downregulated gene were in common 


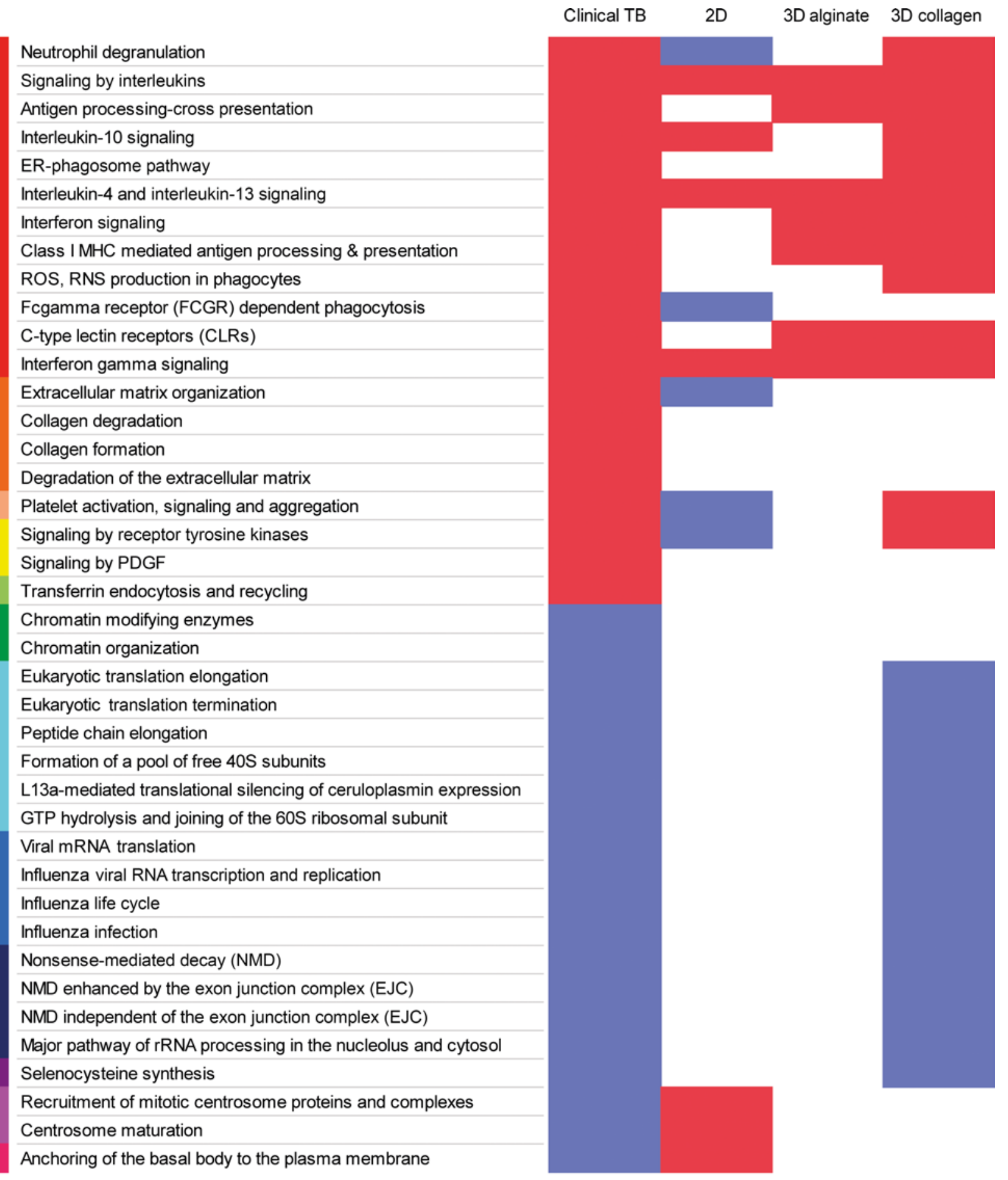

Immune system

Extracellular matrix organization

Hemostasis

Signal transduction

Transport of small molecules
Chromatin organization
Metabolism of proteins
Disease

Metabolism of RNA

Metabolism

Cell cycle

Organelle biogenesis and maintenance

Figure 4. The 3D granuloma model with alginate-collagen matrix most closely reflects clinical TB gene expression. Gene ontology enrichment (ReactomePA program in R, using genes with adjusted $P$ value $<0.05$ ) showing the top 20 REACTOME pathways upregulated and downregulated in human TB granulomas relative to control tissue, according to adjusted $P$ value. Pathways are ordered within in each gene ontology category according to adjusted $P$ value (Clinical TB), and gene ontology category is depicted by color. Significant fold change expression for each pathway denoted across different cell culture models. Red: pathways significantly upregulated in category; blue: pathways significantly downregulated in category (adjusted $P$ value $<0.05$ for each). 
A

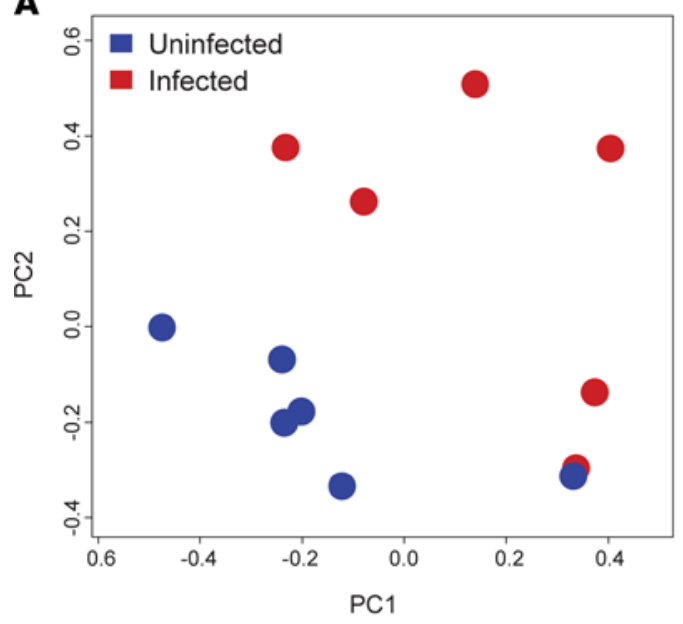

B

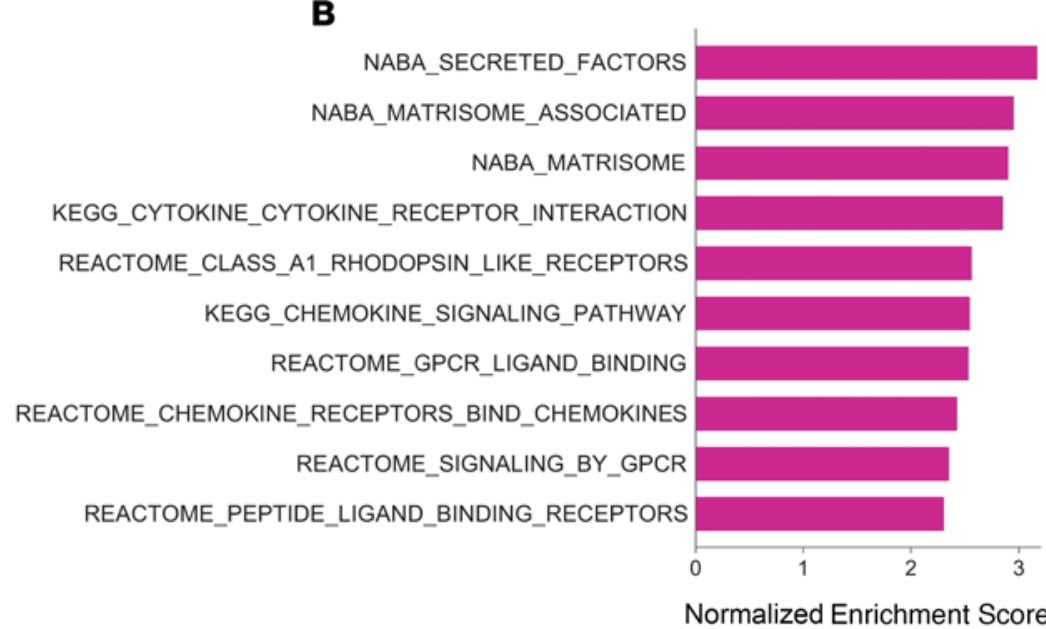

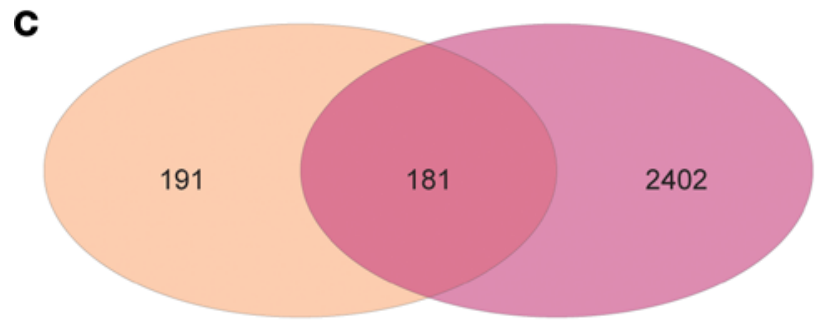

Clinical TB, TB only
3D collagen

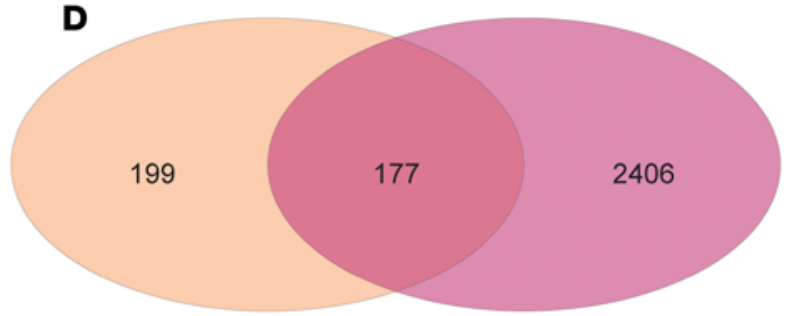

Clinical TB,

TB and sarcoid shared

Figure 5. Mtb infection of PBMCs in the 3D collagen model upregulates multiple genes that overlap with clinical TB specimens. (A) PCA of whole transcriptome data showing differentiation of gene expression between uninfected (blue) and Mtb-infected (red) PBMCs in the 3D collagen model in 6 healthy donors. (B) Top 10 overexpressed canonical pathways according to Normalized Enrichment Score (NES) in Mtb-infected relative to uninfected cells in the 3D collagen model (Gene Set Enrichment Analysis). Adjusted $P$ value $<0.01$. (C) Overlap of genes upregulated uniquely in clinical TB samples (orange) and the 3D collagen model (purple). (D) Overlap of genes upregulated consistently in both TB and sarcoidosis (orange) and the 3D collagen model (purple). Thresholds in clinical samples of $\log _{2}$ fold change $\geq 1.5$ and adjusted $P$ value $<0.05$, and thresholds in the $3 D$ collagen model of $\log _{2}$ fold change $\geq 0.18$ and adjusted $P$ value $<0.05$.

with clinical TB. The 3D collagen model had 482 DEGs, of which 158 upregulated and 7 downregulated genes overlapped with clinical TB samples, and so shared the greatest number of genes.

Gene ontology analysis of DEGs demonstrates that the 3D collagen model reflects the top 20 REACTOME pathways upregulated and downregulated in clinical TB more closely than the other models (Figure 4). The 3D collagen model represents upregulated pathways in clinical TB associated with the immune system, hemostasis, and signal transduction, as well as downregulated pathways relating to metabolism of proteins and RNA. The 3D alginate model has upregulation of several pathways in clinical TB associated with the immune system, but no downregulated pathways overlapping with clinical TB. In contrast, the 2D model correlates with only 4 upregulated pathways in clinical TB, all immune pathways, and demonstrates divergent regulation of several pathways in the opposite direction to the clinical samples. Next, we compared pathways regulated by infection in the 3D collagen model with gene expression profiles in the circulation of patients with TB, and found high overlap and concordance of modulated pathways (Supplemental Figure 14). Therefore, we progressed to our mechanistic studies in the 3D collagen model.

Comparison of clinical samples and the 3D granuloma model identifies potential therapeutic target genes. Initial transcriptomic analysis of uninfected and Mtb-infected PBMCs from 6 healthy donors using principle component analysis in the 3D collagen model demonstrated general separation of groups, with overlap of 1 uninfected and 1 infected microsphere sample that may reflect donor variation (Figure 5A). Using gene set enrichment analysis, the top 10 canonical pathways upregulated in the $3 \mathrm{D}$ collagen model relate to the extracellular matrix and cytokine signaling (Figure 5B), similar to that observed in clinical TB samples (Supplemental Figure 11A).

Next, we compared all significantly upregulated genes in the 3D collagen model, including those with $\log _{2}$ fold change below 1.5 , with the upregulated genes previously identified in clinical TB samples. DEGs from clinical TB were categorized into 2 groups, TB only or shared between TB and sarcoidosis, to determine if targeting pathways unique to TB or shared with sarcoidosis had divergent effects. This identified 181 genes upregulated in clinical $\mathrm{TB}$ and the 3D collagen model (Figure 5C and Supplemental Table 15), and 177 upregulated genes upregulated in both TB and sarcoidosis that were also induced in the 3D collagen model (Figure 5D and Supplemental Table 16).

Selection of candidate host-directed therapy targets. We then applied a systematic approach to host-directed therapy (HDT) target selection, beginning with the 181 genes upregulated in TB only and 
TB only

Upregulated in clinical TB and 3D collagen samples
Intracellular enzyme
Available chemical inhibitor
Inhibitor target specificity
Drug toxicity
Fold change in clinical TB compared to control samples

Fold change in Mtb infected compared to uninfected 3D collagen

Transcript abundance (TPM)

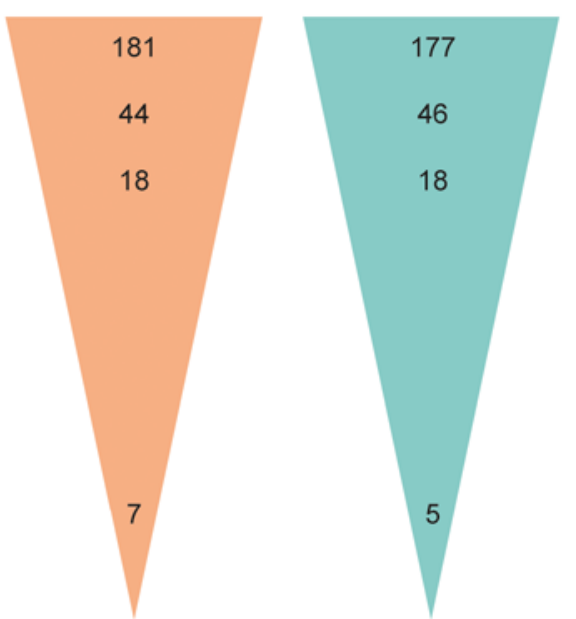

Figure 6. Systematic selection process to identify potential host-directed therapy targets. Upregulated genes that were exclusive to TB granulomas or shared between TB and sarcoidosis granulomas, and that were also upregulated in the 3D collagen model, were systematically refined using the criteria listed. This process identified 12 host-directed therapy targets for experimental inhibition in the 3D collagen model. Thresholds used for initial gene selection were $\log _{2}$ fold change $\geq 1.5$ with adjusted $P$ value $<0.05$ in clinical samples, and $\log _{2}$ fold change $\geq 0.18$ with adjusted $P$ value $<0.05$ in the $3 D$ collagen model.

177 TB-sarcoidosis genes (Figure 6). First, we selected intracellular enzymes as potentially tractable targets for orally available inhibitors, an important consideration for resource-poor settings. We identified 90 enzymes, of which 36 had validated chemical inhibitors available. Target specificity for each compound was reviewed, and inhibitory drugs with multiple targets were excluded. Published drug toxicity data were analyzed, with prioritization of drugs with proven safety in either human or in vivo systems and no evidence of toxicity. The final factors examined were the DEG fold change values in the clinical TB samples and Mtb-infected 3D collagen model, and transcript abundance in the clinical TB samples. This process identified 12 intracellular HDT targets, 7 in the TB only group (CA2, CTSL, FURIN, LRRK2, NAMPT, RIPK2 and SPHK1) and 5 communal to TB and sarcoidosis (FADS2, HK2, HSD11B1, SRC, TYMP).

Sphingosine kinase 1 (SphK1) inhibition suppresses Mtb growth in the $3 \mathrm{D}$ collagen model. To investigate the effect of targeting the pathways identified through this unbiased approach, we studied the effect of inhibitors added to the 3D collagen model at day 1 . Inhibitors were added at a concentration determined by published studies, which are detailed in Supplemental Table 17. Mtb luminescence was observed within 2 hours of drug application, after 24 hours (day 2), and then on days 4, 7, 9, 11, and 14. Inhibitors were supplemented at day 7, after Mtb luminescence readings were taken. PF-543, a specific inhibitor of SphK1 (16), caused the most marked suppression of Mtb growth in a dose-dependent manner, even at the initial reading taken at 2 hours (Figure 7A). SSM3 trifluoroacetate, a furin inhibitor, also demonstrated a dose-dependent reduction in Mtb growth (Figure 7B). To a lesser degree, SC 26196, a FADS2 inhibitor (Supplemental Figure 15C) and 3-Bromopyruvic acid, a HK2 inhibitor (Supplemental Figure 15D), also significantly suppressed Mtb growth. The other 8 inhibitors investigated did not affect Mtb growth (Supplemental Figure 15, A, B, and E-J).

In order to determine if suppression of Mtb growth was due to increased cell death, cytotoxicity studies were performed for PF-543 and the other 11 inhibitors. PF-543 did not significantly increase cytotoxicity (Figure 7C), while brinzolamide, a CA2 inhibitor, caused increased cytotoxicity (Supplemental Figure 15K). To exclude a direct effect on Mtb growth, inhibitors were added to Mtb cultured in Middlebrook 7H9 broth. PF-543 did not affect Mtb growth, whereas SSM3 trifluoroacetate caused significant Mtb growth reduction (Figure 7D). Therefore, suppression of Mtb growth by PF- 543 requires PBMCs, indicating that the effect is most likely via modulating host signaling pathways. SphK1 is a key regulator of the ceramide-sphingosine rheostat, which controls diverse cellular processes, and so the expression of enzymes involved in this pathway was analyzed in the clinical TB samples. SPHK1 and SGPL1, the gene encoding S1P lyase, were upregulated in TB granulomas, while CERS4 (ceramide synthase 4) was downregulated (Figure 7E). The overall impact of these expression changes on the ceramide/sphingosine axis would favor the production of S1P (sphingosine-1-phosphate) and irreversible degradation by S1P lyase, resulting in concurrent depletion of ceramide stores.

SphK1 blockade modulates intracellular acidification and inflammatory mediator secretion. To explore this phenomenon further, we first studied K6PC-5, a specific activator of SphK1 (17). K6PC5 increased Mtb growth within the 3D collagen model, in contrast to suppression by the inhibitor PF-543 (Figure 8A), confirming SphK1 activity favors Mtb growth. Colony counting confirmed that reduced luminescence in PF-543-treated cells was due to bacterial killing (Figure 8B). We observed the effect of PF-543 as early as 2 hours after the addition of PF-543, and we hypothesized that the mechanism may center on phagolysosomal fusion, which is delayed in Mtb-infected cells (18). We studied changes in intracellular $\mathrm{pH}$ by staining cells with pHrodo, which increases fluorescence at low $\mathrm{pH}$ (19). Mtb infection reduced fluorescence of monocytes compared with uninfected cells, consistent with inhibition of phagolysosomal fusion, while PF-543 normalized fluorescence to that of uninfected cells (Figure $8 \mathrm{C}$ ). In contrast, Bafilomycin A1, a lysosomal inhibitor, did not cause an increase in fluorescence in infected monocytes, and K6PC-5 did not have an effect (Supplemental Figure 16A). We 

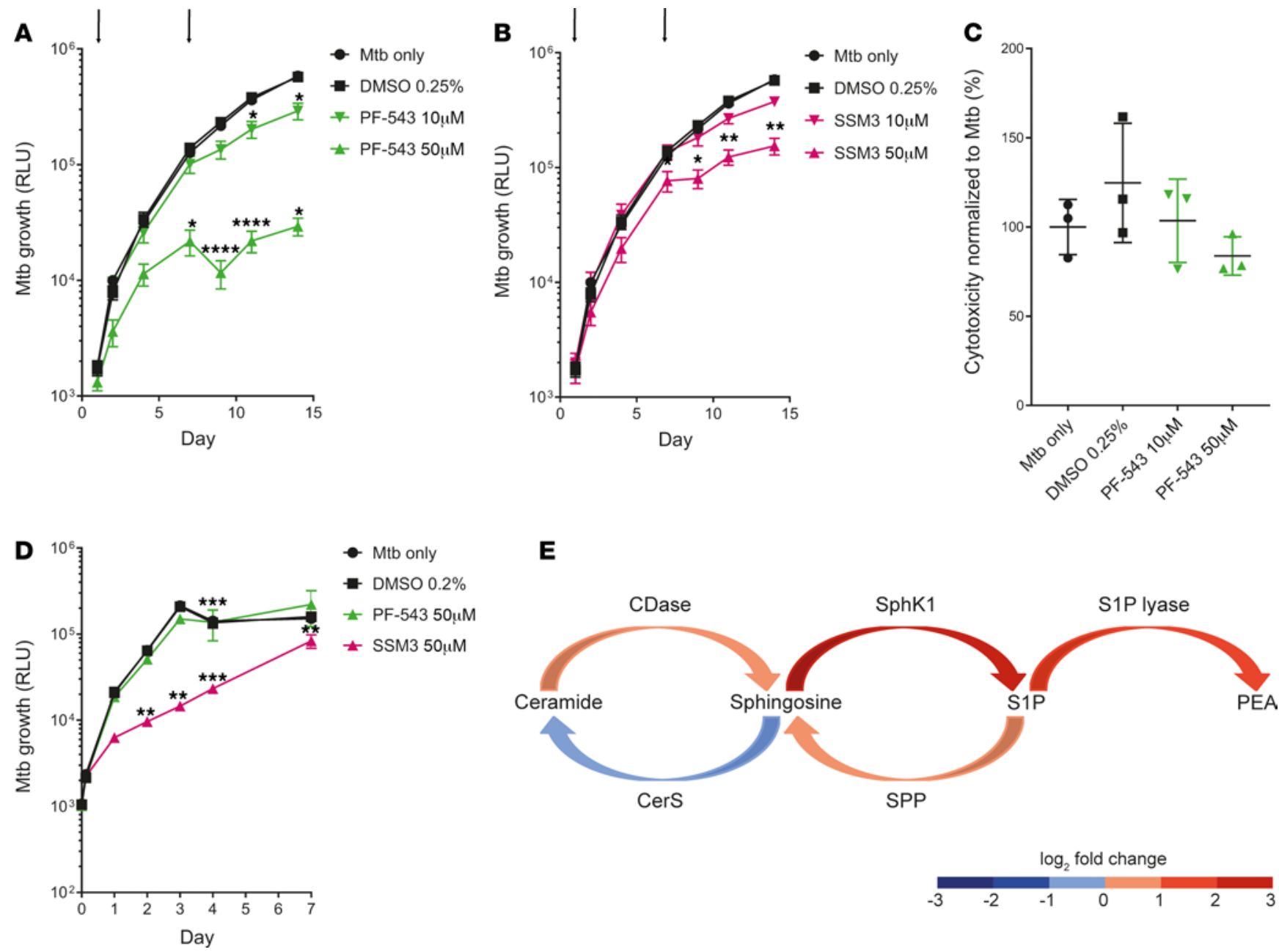

Figure 7. SphK1 inhibition suppresses Mtb growth. (A) Mtb growth in 3D collagen model measured by luminescence (relative light units [RLU]): untreated (black circles), treated with DMSO (black squares), SphK1 inhibitor PF-543 (green triangles). Black arrows indicate drug addition on days 1 and 7. Analysis was by 2-way ANOVA; error bars indicate SD. (B) Mtb growth in 3D biomimetic model cultures untreated (black circles), or treated with DMSO (black squares) or furin inhibitor SSM3 trifluoroacetate (pink triangles). Black arrows indicate drug addition. Analysis was by 2-way ANOVA; error bars indicate SD. The same controls are presented in Figure 7, A and B and Supplemental Figure 15, A-J, and all conditions were compared simultaneously against the control by Dunnett's multiple comparison test. (C) Relative cytotoxicity in 3D collagen model with SphK1 inhibition: untreated (black circles), or treated with DMSO (black squares) or SphK1 inhibitor PF-543 (green triangles). Horizontal bars indicate mean, error bars indicate SD. (D) Mtb growth in Middlebrook 7 H9 broth culture: untreated (black circles), or treated with DMSO (black squares), SphK1 inhibitor PF-543 (green triangles), or furin inhibitor SSM3 trifluoroacetate (pink triangles). Analysis was by 2-way ANOVA; error bars indicate SD. (E) Graphical representation of changes in sphingolipid signaling pathway genes in TB granulomas relative to control lymph nodes (adjusted $P$ value $<0.05$ for SphK1 and S1P lyase, otherwise more than 0.05). CDase, ceramidase; CerS, ceramide synthase; S1P, sphingosine-1-phosphate; SPP, sphingosine-1-phosphate phosphatase; PEA, phosphoethanolamine. ${ }^{*} P<0.05,{ }^{* *} P<0.01,{ }^{* * *} P<0.001,{ }^{* * *} P<0.0001$.

performed kinetic analysis of intracellular $\mathrm{pH}$, to determine if the reduced $\mathrm{pH}$ was maintained, and demonstrated that increased fluorescence in PF-543-treated cells persisted for 40 minutes when analyzed by both the pHrodo assay (Figure 8D) and also LysoSensor, a second intracellular pH assay (Supplemental Figure 16B). PF-543 had such a rapid effect that fluorescence already diverged in the time between addition of PF-543 to the cells and the first reading, and fluorescence increased further before stabilizing. SphK1 inhibition also suppressed secretion of inflammatory mediators upregulated in TB identified in the clinical sample analysis, such as CCL2 (Figure 8E) and MMP-1 (Figure 8F). Finally, to investigate the translational potential of targeting SphK1 in patients and to identify the cellular origin, we performed immunohistochemical analysis of lung biopsies from patients with TB. Epithelioid macrophages and multinucleate giant cells within TB granulomas were immunoreactive for SphK1 (Figure 8, G-I). SphK1 expression was observed in a subset of granuloma macrophages, and was very rarely observed in macrophages distal to the granuloma or in normal lung tissue.

\section{Discussion}

Through a combination of transcriptomic analysis of treatment-naive clinical lymph node samples alongside a biomimetic TB granuloma model, this study explored processes dysregulated in human TB and identified novel potential therapeutic pathways. The primary pathways dysregulated in TB related to excessive inflammation and extracellular matrix degradation. SphK1 emerged as an important regulator, with inhibition of SphK1 leading to rapid Mtb death and suppression of inflammatory mediator secretion. Furthermore, 

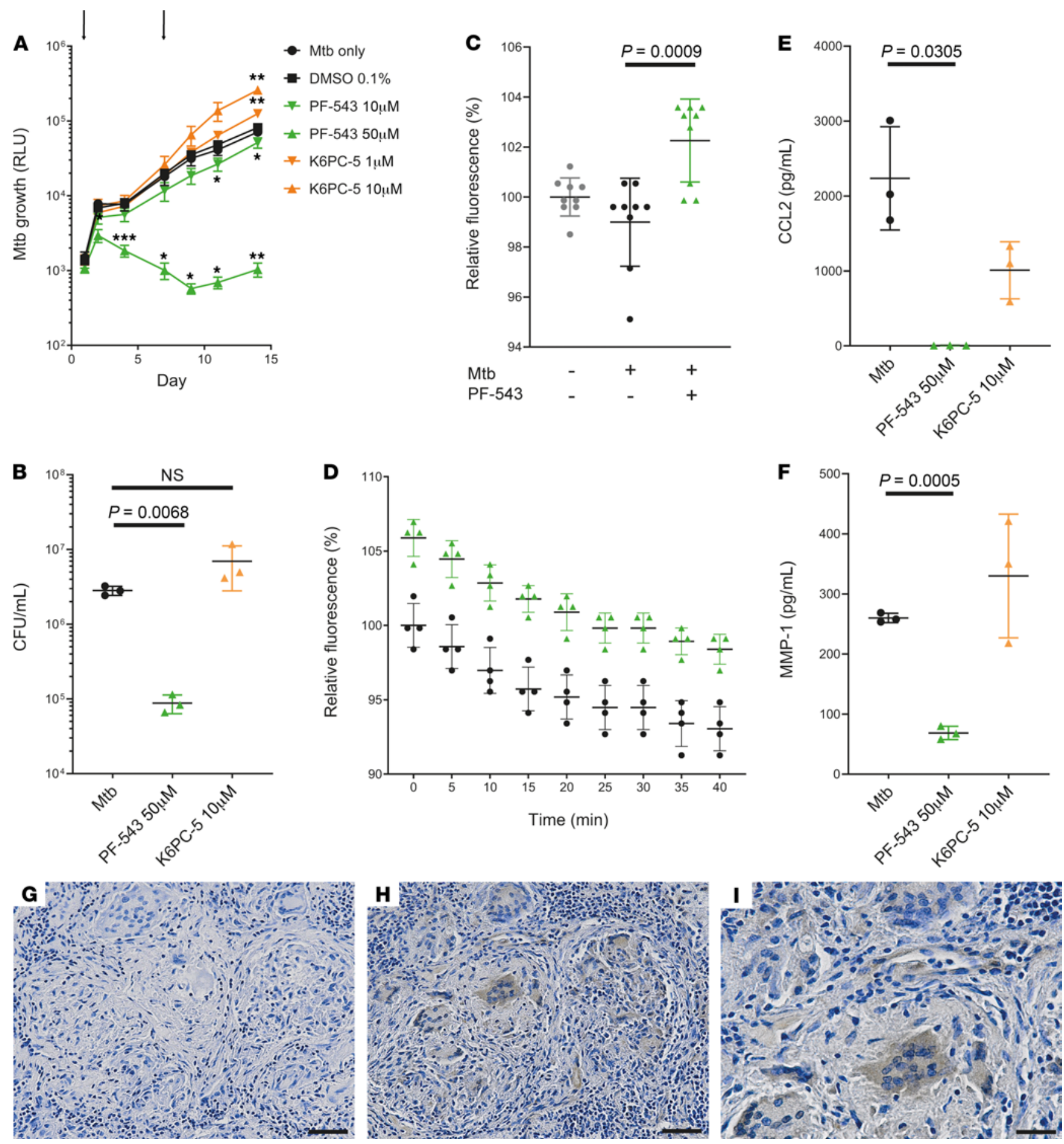

Figure 8. SphK1 regulates the host-pathogen interaction in TB and is expressed in human granulomas. (A) Mtb growth detected in 3D collagen model measured by luminescence (RLUs): untreated (black circles), DMSO (black squares), SphK1 inhibitor PF-543 (green triangles), and SphK1 activator K6PC-5 (orange triangles). Black arrows: drug addition on days 1 and 7. Analysis was by 2-way ANOVA; error bars indicate SD. (B) Mtb CFUs from PBMCs in 3D collagen microspheres, decapsulated and plated on Middlebrook 7H11 agar: DMSO 0.1\% (black circles), SphK1 inhibitor PF-543 (green triangles), SphK1 activator (orange triangles). Difference analyzed by paired $t$ test. Horizontal bars indicate mean, error bars indicate SD. (C) Relative fluorescence signal in human monocytes stained with pHrodo measured 5 minutes after treatment with DMSO $0.1 \%$ and Mtb infection with or without concurrent addition of 50 $\mu \mathrm{M}$ PF-543. Increased fluorescence signal indicates lower $\mathrm{pH}$. Normalized data from 2 separate donors, analyzed by paired $t$ test. Horizontal bars indicate mean, error bars indicate SD. (D) Relative fluorescence signal in human monocytes stained with pHrodo, measured at 5 minute intervals after Mtb infection for 40 minutes, treated with DMSO $0.1 \%$ (black circles) or $50 \mu \mathrm{M}$ PF-543 (green triangles). Normalized data shown from one donor. Horizontal bars indicate mean, error bars indicate SD. (E) Secretion of CCL2 and (F) MMP-1 from 3D collagen model into tissue culture media on day 7 after Mtb infection: treated with DMSO 0.1\% (black circles), SphK1 inhibitor PF-543 (green triangles), or SphK1 activator (orange triangles). Analyzed by paired $t$ test. Horizontal bars indicate mean, error bars indicate SD. (G, H, and I) Immunohistochemistry of human lung TB granulomas stained with SphK1 antibody. No reactivity is observed without antibody (G), while SphK1 expression is demonstrated in a subset of macrophages and multinucleate giant cells (brown stain, $\mathbf{H}$ and $\mathbf{I}$ ). Scale bars: $50 \mu \mathrm{m}(\mathbf{G}$ and $\mathbf{H}), 25 \mu \mathrm{m}(\mathrm{I}) .{ }^{*} P<0.05,{ }^{* *} P<0.01$. 
SphK1 is overexpressed in human TB granulomas. Overall, our approach of integrated analysis of human clinical tissue alongside a biomimetic 3D model provides a translational pipeline to identify new therapies and dissect their underlying mechanism of action.

TB shares many histological and clinical features with sarcoidosis, and so we included sarcoidosis as an important noninfectious granuloma comparator group. In the preantibiotic era, one-third of those with active pulmonary TB spontaneously recovered (8), while sarcoidosis regresses without treatment in two-thirds of individuals $(5,20)$, indicating a fine balance between disease persistence and granuloma resolution. Our RNAseq analysis of granulomas isolated from TB and sarcoidosis biopsies provides deep insight into disease mechanisms, identifying hundreds of differentially expressed genes compared with control lymph nodes. Many dysregulated pathways are shared between the 2 diseases and fall into expected categories, such as chemokine and interferon signaling (21) and extracellular matrix organization (20). However, some pathways were relatively unexpected, such as lysosomal pathways, which have not previously been identified as being important in sarcoidosis (20). We observed overlap between the TB signature and autoimmune diseases such as rheumatoid arthritis, consistent with emerging evidence of shared underlying pathways (22). Of note, while many pathways were shared between TB and sarcoidosis, they were often dysregulated to a higher degree in TB, implying that the degree of immune activation may be critical in determining whether the outcome is protective or deleterious to the host (23). Identification of gene clusters specific to each disease permitted unique insight into the underlying pathophysiological mechanisms that led to the differential outcomes, despite the extensive overlap in expression patterns and histological similarities.

A defining characteristic of human TB is lung destruction and cavitation, leading to morbidity, mortality, and transmission. Matrix metalloproteinases play a central role in lung matrix turnover (24). MMP1, also known as interstitial collagenase, is among the top 10 upregulated genes according to fold change in TB compared with control samples, and degrades lung extracellular matrix in TB (25). Furthermore, MMP1 was the most upregulated gene in TB compared with sarcoidosis, with the highest fold change $\left(\log _{2}\right.$ fold change 7.6) of all differentially expressed genes, and notably, TB causes much more extensive lung matrix destruction than sarcoidosis. The mouse lacks a functional orthologue of MMP1 (26), potentially explaining why immunopathology differs in the mouse model of TB. Prior studies from our group infecting mice expressing a human MMP-1 transgene demonstrated a critical role for MMP1 in TB immunopathology $(25,27)$. However, therapeutic targeting of MMPs in animal models have provided mixed results, with inhibitor monotherapy paradoxically worsening pathology $(28,29)$, while beneficial when combined with antibiotics (30), suggesting efficacy requires concurrent MMP inhibition and bacterial killing. Supporting this, a phase 2 clinical trial has shown that adjunctive doxycycline, a broad spectrum MMP inhibitor, alongside standard antibiotic treatment of patients with pulmonary $\mathrm{TB}$, reduces MMP expression and cavity volume (31).

Correlation analysis identified a cluster of 7 genes that were TB unique, including MMP1 and SLC11A1, CCL7, CCL8, LGALS17A, FAM124A, and OLR1, suggesting that these genes play a central role in TB pathogenesis. Notably, SLC11A1 (NRAMP1) polymorphisms were the first to be linked to TB susceptibility more than 20 years ago (32). SLC11A1 exerts pleiotropic effects on macrophage function that will increase inflammation, including enhanced expression of MHC class II, cytokines such as TNF- $\alpha$ and IL-1 $\beta$, and inducible nitric oxide synthase (33). The high expression of chemokines $C C L 7$ (MCP3) and CCL8 (MCP2) supports the emerging concept that excessive monocyte recruitment is harmful in TB (34). LGALS17A and FAM124A may augment inflammation through NFKB activation (35), though their roles are less well defined. OLR1 can regulate foamy macrophage formation, a typical feature of the human TB granuloma (36), and contributes to excessive inflammation in atherosclerosis and myocardial ischaemia $(37,38)$. Moreover, OLR1 activation induces epigenetic reprogramming in macrophages (39), suggesting trained immunity may contribute to the hyperinflammatory state observed in TB. Considering this cluster of 7 TB-specific genes together implies that dysregulated inflammation is a sequential process, whereby excessive monocytes are recruited to the granuloma (CCL7 and CCL8), reprogrammed to a hyperinflammatory phenotype (OLR1), further propagating inflammation (SLC11A1) and $\mathrm{NF \kappa B}$ activation (FAM124A), resulting in extracellular matrix degradation (MMP1), cavity formation, and transmission.

Differential gene analysis further informs comparisons between sarcoidosis and TB, whereby the most significantly dysregulated biological process was the cytokine-mediated signaling pathway, comprising of upregulated genes including SPHK1, MMP1, and both proinflammatory and immunoregulatory cytokines. Other upregulated processes in TB compared with sarcoidosis centered on angiogenesis and extracellular matrix degradation. The only cellular component significantly downregulated in TB relative to sarcoidosis is the lysosome, which is critical in antigen processing (40). The pathways that we identified only show partial overlap with RNAseq analysis of circulating immune cells from TB patients (41, 42), demonstrating the importance of studying tissue in investigating local pathogenic mechanisms. Consistent with this observation, the majority of genes identified as potential HDT targets were also upregulated in granulomas from patients undergoing surgery for TB treatment failure analyzed by microarray (43). Our data set is unique in analyzing human TB biopsies pretreatment with the microenvironment sampled by laser capture microdissection, without the confounder of treatment. However, our samples were from lymph nodes as opposed to the lung parenchyma, and so compartment-specific differences are likely. An ideal comparator would be lung, lymph node, and blood from the same patient taken before treatment, with each tissue microenvironment spatially analyzed. However, recruiting such a cohort may be logistically impossible.

Evaluation of the potential therapeutic targets identified from the clinical samples requires study in an appropriate cell culture model. Bioinformatic analysis of standard 2D cell culture, 3D alginate, and 3D collagen models identified that the 3D collagen model has the most overlap with clinical TB samples, consistent with the emerging importance of 3D models incorporating extracellular matrix to understand biological phenomena $(44,45)$. Such biomimetic models are advancing the field of cancer immunotherapy (46), and we demonstrate similar applicability for infectious disease. Previously, our group has shown that the 3D collagen model can identify harmful effects of an excessive immune response in TB (14), dissect the mechanism whereby PD-1 inhibition leads to TB reactivation (47), and study the protective effect of IL-17 (48). 
To translate the bioinformatic analysis toward clinical application, we focused on intracellular kinases as potential therapeutic targets, as orally available signaling pathway inhibitors have proven clinical utility, such as aspirin, indomethacin, and imatinib. Within the 358 upregulated genes in TB shared with the 3D collagen model, many additional potential therapeutic targets have not yet been investigated. A systematic screening approach demonstrated that SphK1 inhibition suppresses Mtb growth within the granuloma model independent of an effect on Mtb. SphK1 is a critical enzyme in the sphingosine-ceramide cellular rheostat, which modulates diverse cellular processes (49). SphK1 phosphorylates sphingosine to sphingosine-1-phosphate (S1P), which is then irreversibly degraded by S1P lyase. S1P is a signaling molecule with a complex mechanism of action, regulating diverse processes including transcription, angiogenesis, atherosclerosis, apoptosis, and autophagy (50). By diverting the sphingolipid pathway away from S1P synthesis, SphK1 inhibition will concurrently increase cellular ceramide levels (16).

SphK1 inhibition suppressed Mtb growth surprisingly rapidly, and this suggested that the mechanism may be through increasing phagolysosomal fusion, which Mtb inhibits as part of its intracellular survival strategy $(18,51)$. Consistent with this conclusion, SphK1 inhibition normalizes intracellular $\mathrm{pH}$, and previous studies have shown that ceramide accumulation increases phagolysosomal fusion. Ceramide accumulates and causes Mtb killing by regulating actin nucleation (52), although a report from an alternative 2D model system differs from this conclusion (53). Taking these findings into account with our observations suggests that directing sphingosine metabolism to S1P, and away from ceramide, is part of Mtb's intracellular survival strategy. However, an emerging concept is that diverse endotypes exist in TB (54), and so it may emerge that targeting the sphingosine pathway will work in some, but not all, clinical manifestations of TB. SphK1 inhibition has significant potential, as activating innate killing will circumvent drug resistance mechanisms in TB. However, because mice do not express a functional orthologue of MMP-1 (26), the full effect of SphK1 inhibition on TB immunopathology will need to be assessed in more complex animal model systems prior to human studies.

In summary, a tissue RNAseq approach comparing 2 granulomatous conditions provided novel insight into the underlying disease mechanisms in human TB. Integrating the clinical analysis with a 3D biomimetic cellular model to screen for host-directed therapy targets identified SphK1 inhibition as a potential approach to complement current antibiotic therapy. Enhancing host cell mycobacterial killing through this strategy may shorten the duration of therapy and improve outcomes in disease.

\section{Methods}

Clinical sample selection. Clinical samples were selected from adult patients undergoing a mediastinal or neck lymph node biopsy, with all biopsies performed at University Hospital Southampton NHS Foundation Trust. Biopsies were formalin-fixed and paraffin-embedded (FFPE) immediately after the procedure. Each specimen had typical histological appearance for each disease and was verified by a consultant histopathologist. Individual patients' medical records were reviewed to exclude confounders, such as ensuring patients were nonsmokers at the time of biopsy and had not received antituberculous or immunosuppressant therapy prior to biopsy excision. Consequently, all biopsy samples are from treatment-naive patients. To maximize efficiency of library chip use for RNAseq, a total of 24 samples were planned. Eleven samples from each clinical group were initially processed to provide an excess of samples and optimize chances of obtaining adequate RNA yield for RNAseq (Supplemental Figure 1).

TB biopsies were culture positive for drug-sensitive Mtb and had typical TB caseating granulomas, taken from either mediastinal or neck lymph nodes. All patients had negative HIV serology and no significant comorbidities. The patients' mean age was 36 years old ( 23 to 56 years old); there were 6 males and 5 females.

Sarcoidosis biopsies had classical sarcoidosis granulomas in mediastinal lymph nodes. All patients had negative Mtb culture results and no positive HIV serology (HIV serology was negative or not performed). Three patients had comorbidities, specifically mild ulcerative colitis, chronic kidney disease stage IV, and diabetes mellitus type 2, though none had received immunosuppressant therapy. The patients' mean age was 59 years old (39 to 76 years old); there were 4 males and 7 females.

Reactive lymph nodes from patients with newly diagnosed cancer were selected as control samples, with no evidence of metastases. No patients had positive Mtb culture or positive HIV serology. The patients' mean age was 57 years old (26 to 70 years old); there were 7 males and 4 females.

Laser capture microdissection of clinical biopsies. All instruments and tools were cleaned with RNaseZap RNase Decontamination Solution (RNaseZap RNase decontamination wipes, Life Technologies). FFPE biopsy blocks were cut with a Leica RM2135 microtome. After discarding the first $20 \mu \mathrm{m}$, sections of $10 \mu \mathrm{m}$ thickness were cut, floated in RNase-free water (UltraPure DNase/RNase-Free Distilled Water, Life Technologies) at $45^{\circ} \mathrm{C}$, mounted on PEN (polyethylene naphthalate) membrane glass slides (Life Technologies), and dried in a drying oven at $37^{\circ} \mathrm{C}$ overnight. To dewax, the sections were twice immersed in Xylene (Arcturus Paradise Plus Staining components, Life Technologies) for 5 minutes at a time. No staining of tissue was required. To remove the Xylene, sections were twice immersed in 100\% EtOH (Absolute 200 Proof, Molecular Biology Grade, Fisher BioReagents) for 1 minute at a time, then left to air dry for up to 1 hour. The laser capture microdissection instrument (Zeiss PALM MicroBeam) was optimized (energy 66, speed $10 \%$, and focus 75). From each biopsy, sequential areas were captured until a total of $8 \mathrm{~mm}^{2}$ had been dissected, acquiring $4 \mathrm{~mm}^{2}$ per capture tube (Zeiss AdhesiveCap 500 opaque). In initial optimizations, this area yielded sufficient RNA for subsequent sequencing. Thereafter, each tube sample was exposed to $100 \mu \mathrm{L}$ digestion buffer, then $4 \mu \mathrm{L}$ protease, and the tubes incubated in heat blocks at $50^{\circ} \mathrm{C}$ for 30 minutes, and then $80^{\circ} \mathrm{C}$ for 14 minutes to inactivate the protease. Each tube was subsequently frozen at $-80^{\circ} \mathrm{C}$. Digestion buffer and protease were from RecoverAll Total Nucleic Acid Isolation Kit for FFPE (Invitrogen).

Total RNA extraction from clinical samples. Micro Filter Cartridges were from RNAqueous-Micro Total RNA Isolation Kit (Invitrogen). Samples were thawed at room temperature. Isolation buffer was mixed with each sample and up to $500 \mu \mathrm{L}$ loaded on to a prepared Micro Filter Cartridge Assembly. As per kit instructions, each Micro Filter Cartridge Assembly was centrifuged, Wash 1 Solution and Wash 2/3 Solution used to isolate total RNA, and DNase mix then applied. RNase-free water (UltraPure DNase/RNase-Free Distilled Water, Life Technologies) was used for elution at a volume of $12 \mu \mathrm{L}$, 
and samples stored at $-80^{\circ} \mathrm{C}$. RNA quality and quantity were checked by Agilent 2100 Bioanalyzer (Agilent Technologies) and Qubit 2.0 Fluorometer (Thermo Fisher Scientific), respectively.

RNA sequencing of clinical samples. Sequencing of clinical samples was performed at the Kaminski laboratory, Department of Pulmonary, Critical Care, and Sleep Medicine, Yale School of Medicine. Ion Torrent sequencing was used, and all reagents were from Life Technologies. Twenty-four samples were prepared to make cDNA libraries using Ion AmpliSeq. Human Gene Expression kit (Ion Torrent) using $15 \mathrm{ng}$ total RNA. As per the manufacturer's instructions, the Ion PI Hi-Q Chef Kit on the Ion Chef instrument was used for library construction, assembling 2 chips from 8 diluted samples at a time. The Ion Proton System was used as per the manufacturer's instructions to sequence RNA. Total RNA of $15 \mathrm{ng}$ per sample was used to produce a mean average of 18 million single-end, approximately 100 base pair reads per sample and transferred in FASTQ format (GEO accession code GSE174443).

$P B M C$ isolation from human blood. PBMC isolation from single leukocyte donor cones (National Health Service Blood and Transfusion, Southampton, United Kingdom) was performed by density gradient centrifugation over Ficoll-Paque (GE Healthcare Life Sciences). Blood donors were healthy individuals with negative HIV and hepatitis B/C serology living in a low TB endemic area. Six blood donors were studied in each of the 3 cell culture models. PBMCs were cultured in RPMI 1640 with 10\% fetal calf serum, L-glutamine, ampicillin, and kanamycin.

M. tuberculosis culture. Bioluminescent $\mathrm{H} 37 \mathrm{Rv}$ (55) was used in all experiments, and cultured in Middlebrook 7H9 medium supplemented with $10 \%$ ADC, $0.2 \%$ glycerol, and $0.02 \%$ Tween 80 (BD Biosciences) with $25 \mu \mathrm{g} / \mathrm{mL}$ kanamycin. Cultures at $1 \times 10^{8} \mathrm{CFU} / \mathrm{ml} \mathrm{Mtb}$ (optical density $=0.6$ ) were used for all experiments at a MOI of 0.1, except in pHrodo experiments where a MOI of 1 was used for monocyte infection. Luminescence was measured with a GloMax 20/20 single tube luminometer (Promega). Mtb-infected PBMCs were released from 3D collagen microspheres by decapsulation and Mtb was cultured after serial dilution on Middlebrook 7H11 agar supplemented with $10 \%$ OADC, $0.2 \%$ glycerol, and $0.4 \mu \mathrm{g} / \mathrm{mL}$ amphotericin.

Microencapsulation of cells. Microspheres were generated using an electrostatic generator (Nisco) as previously described (56). To summarize, PBMCs were infected overnight with Mtb in a $250 \mathrm{~cm}^{2}$ flask. The PBMCs were then detached, pelleted, and mixed with $1.5 \%$ sterile alginate (Pronova UP MVG alginate, Nova Matrix), with or without $1 \mathrm{mg} / \mathrm{mL}$ collagen (Advanced BioMatrix), at a final concentration of $5 \times 10^{6}$ cells $/ \mathrm{mL}$. The cell-alginate suspension was injected into the electrostatic generator where microspheres formed in an ionotropic gelling bath of $100 \mathrm{mM} \mathrm{CaCl}_{2}$ in $\mathrm{HBSS}$. After twice washing with $\mathrm{HBSS}$ with $\mathrm{Ca}^{2+} / \mathrm{Mg}^{2+}$, microspheres were incubated at $37^{\circ} \mathrm{C}$ in RPMI 1640 medium containing L-glutamine, ampicillin, kanamycin, and $10 \%$ human $\mathrm{AB}$ serum. Microspheres were either placed into Eppendorf tubes and randomly allocated to different experimental conditions with subsequent Mtb luminescence measured, or placed into a 96-well plate with conditions in triplicate according to a predetermined template for cytotoxicity studies on day 7. Supernatant was collected from Eppendorf tubes on day 7 after luminescence was measured. Time points described refer to days after infection.

Microsphere decapsulation and cell lysis. Decapsulation solution was $55 \mathrm{mM}$ sodium citrate and $5 \mathrm{mM}$ EDTA with HBSS, with $\mathrm{pH}$ adjusted to 7.4 by adding $5 \mathrm{M} \mathrm{HCl}$. The decapsulation solution was warmed to $37^{\circ} \mathrm{C}$ and on day 4 applied to microspheres after washing with HBSS. Once decapsulated, the cells were centrifuged at $2000 \mathrm{~g}$ for 8 minutes at $4^{\circ} \mathrm{C}$. The same centrifugation was used to pellet PBMCs in 2D culture on day 4 . After centrifugation, cell pellets were resuspended in QIAzol Lysis Reagent (Qiagen) and stored at $-80^{\circ} \mathrm{C}$.

Total RNA extraction from cell culture models. Samples were thawed on ice, and $200 \mu \mathrm{L}$ chloroform added per $1 \mathrm{~mL}$ of sample. Samples were shaken for 15 seconds, incubated for 3 minutes at room temperature, then centrifuged at $12,000 \mathrm{~g}$ for 15 minutes at $4^{\circ} \mathrm{C}$. The upper aqueous layer was extracted. Then manufacturer's instructions were followed (RNeasy Midi Kit, Qiagen), adding 70\% ethanol and shaking. Samples were added to RNeasy Midi columns, and washed with Buffer RW1 and Buffer RPE. Total RNA was eluted with $125 \mu \mathrm{L}$ RNase-free $\mathrm{H}_{2} \mathrm{O}$ and stored at $-80^{\circ} \mathrm{C}$. RNA quality and quantity was checked by Agilent 2100 Bioanalyzer (Agilent Technologies) and NanoDrop ND-1000 UV-Vis Spectrophotometer, respectively.

RNA sequencing of cell culture models. Sequencing was performed by GENEWIZ. Thirty-six samples were sequenced using Illumina HiSeq, with 12 samples per lane. A configuration of $2 \times 150$ base pairs per lane was used, and a mean average of 31 million paired end reads generated and provided in FASTQ format (submitted to GEO accession code 174566).

Bioinformatics pipeline for RNAseq analysis. The same bioinformatics pipeline was used to analyze the RNAseq data from clinical samples and cell culture samples. FastQC software executed quality control checks. Alignment was performed using kallisto software (version 43.1), with sequence based bias correction (57). The human transcriptome and the transcriptome of the Mtb strain $\mathrm{H} 37 \mathrm{Rv}$ were used as index transcriptomes to which alignment was performed. The tximport program (version 1.10.1) imported the reads aligned by kallisto (58), and ensembldb program (version 2.6.8) annotated them to gene level (59). Sleuth was also used to annotate the data aligned by kallisto, to confirm similar results (60). Intersample normalization was performed using TMM (trimmed mean of $\mathrm{M}$ value) normalization (edgeR, version 3.24.3). Unless otherwise stated, all analyses were performed and figures generated in $\mathrm{R}$ environment including principal component analysis, outlier detection, heat map, and volcano plot generation. An interactive tool was used for comparing lists with Venn diagrams, https://bioinfogp.cnb.csic.es/tools/venny/ index.html, and Venn diagrams were generated in R environment.

Differential gene analysis was performed using limma with its voomWithQualityWeights function (version 3.38.3). Voom transformation is required for limma to use the Gaussian distribution (61). A Benjamini-Hochberg FDR-adjusted $P$ value of less than 0.05 was applied. Filter values were optimized to yield the highest number of differentially expressed genes across the study cohort.

TMM-normalized, voom-transformed data were taken forward for gene coexpression analysis. Graphia Professional (version 2.1) (62) used the Markov Cluster Algorithm to perform correlation analysis with Pearson's $r \geq 0.83$, MCL inflation value of 1.7, preinflation value of 3 , scheme value of 6 , and smallest cluster allowed of 5 genes. GraphPad Prism 8 was used to plot the expression intensity of clusters.

Gene sets from the Molecular Signatures Database (MSigDB) (version 7.0) were used (63). Two methods were employed: fast gene set enrichment analysis (fgsea) program (version 1.8.0) was used to 
generate Normalized Enrichment Scores; and Ensemble of Gene Set Enrichment Analyses (EGSEA) using 11 prominent algorithms (camera, gage, globaltest, gsva, ora, padog, plage, roast, safe, ssgsea, zscore) to visualize pathways and generate fold change values identified by gene set enrichment analysis (version 1.10.1) (64).

Gene ontology enrichment analysis for differentially expressed genes was performed using ReactomePA software program (version 1.26.0) (65), while ToppFun functional enrichment in ToppGene suite (66) was used to analyze clusters identified on correlation analysis. An adjusted $P$ value of less than 0.05 was taken as significant.

Chemical inhibitors and supplementation. The following chemical inhibitors were from ApexBio: Brinzolamide (catalog A4359); 3-Bromopyruvic acid (catalog B7922); GNE-7915 (catalog B3605); KX2-391 (catalog B2282); PF-543 (catalog A3717); and Tipiracil (catalog A3875). The following chemical inhibitors were from BioTechne Ltd: CHS 828 (catalog 6753); GSK 583 (catalog 6480); PF 915275 (catalog 3291); SC 26196 (catalog 4189); SID 26681509 (catalog 3625); SSM3 trifluoroacetate (catalog 5253), and Bafilomycin A1 (catalog 1334). K6PC-5 (catalog SML1709) was from Sigma-Aldrich. Both 3-Bromopyruvic acid and Tipiracil were reconstituted in water, and all other chemicals reconstituted in DMSO. Chemicals were sterilized by filtration through a $0.22 \mu \mathrm{M}$ Durapore membrane (Merck Millipore).

Cell toxicity assay. CytoTox-Glo Cytotoxicity Assay (Promega) was performed on day 7 by measuring the activity of intracellular dead cell protease using a luminogenic peptide substrate AAF-Glo Substrate as per manufacturer's instructions.

Monocyte isolation from human blood. Monocytes were isolated from human blood donors as per PBMC isolation described above. Thereafter, the PBMC pellets were resuspended in RPMI 1640 with ampicillin and L-glutamine, and separated using a hyperosmolar Percoll gradient. Monocytes were then used in the $\mathrm{pH}$ indicator assays, and infected with Mtb at MOI of 1.

Intracellular $\mathrm{pH}$ indicator assays. Fresh monocytes were stained with pHrodo iFL Green STP Ester amine reactive dye (Thermo Fisher Scientific) by making $10 \mathrm{mM}$ stock solution with DMSO and then diluting with RPMI and $2 \mathrm{mM}$ EDTA to give $10 \mu \mathrm{M}$ at $\mathrm{pH}$ 7.4. After isolation, monocytes were stained with pHrodo and incubated at $37^{\circ} \mathrm{C}$ for 30 minutes, before washing with RPMI and 2 mM EDTA and resuspending in RPMI 1640 with 10\% human AB serum, ampicillin, and L-glutamine. Monocytes were transferred to a 96-well plate for overnight incubation at $37^{\circ} \mathrm{C}$. Mtb infection and addition of compounds occurred in quick succession, and fluorescence readings were taken every 5 minutes for 40 minutes using GloMax Discover (Promega). A second intracellular pH assay, LysoSensor Green DND-189 (Thermo Fisher Scientific) was used with frozen monocytes. Monocytes were thawed and transferred to a 96 -well plate for incubation at $37^{\circ} \mathrm{C}$ for 2 hours, then infected with Mtb for a further 1 hour at $37^{\circ} \mathrm{C}$ before the addition of compounds and $2 \mu \mathrm{M}$ LysoSensor in quick succession. Fluorescence readings were taken every 5 minutes for 40 minutes using GloMax Discover (Promega). Quadruplicate conditions and a minimum of 3 separate donors were used for each $\mathrm{pH}$ assay.

Immunohistochemistry of FFPE tissue. Immunohistochemical staining of FFPE lung tissue from TB and control patients was performed using $4 \mu \mathrm{m}$ sections, using a previously optimized antiSphK1 antibody (ab61148, abcam), and the avidin-biotin-peroxidase technique.
Luminex analysis. Samples were sterilized by filtration through a $0.22 \mu \mathrm{m}$ Durapore membrane (Merck Millipore). Concentrations of cytokines (Thermo Fisher Scientific) and MMPs (R\&D Systems) were measured using a Bioplex 200 platform (Bio-Rad) according to the manufacturer's protocol.

Statistics. Experiments were performed on at least 3 occasions using PBMCs from 3 separate donors with triplicate conditions. Colony forming units were counted from 2 separate donors in triplicate. Data presented are from a representative donor and include the mean and SD, and are consistent across donors. Analysis was performed in GraphPad Prism 8. Student's $t$ test was used to compare pairs. ANOVA with Dunnett's correction for multiple comparisons was used for groups of 3 or more, where control was DMSO for all chemicals reconstituted in DMSO, and control was Mtb only for those reconstituted in water.

Study approval. Analysis of PBMCs was approved by the National Research Ethics Service Committee South Central-Southampton A (ethics approval reference 13/SC/0043). All donors gave written informed consent. Histological analysis of biopsy samples was approved by the Southampton Research Biorepository (ethics approval reference 12/NW/0794 SRB04_14). Lymph node biopsy tissue was taken as part of routine clinical care for diagnostic purposes, and surplus tissue was analyzed in this study at a later date. The ethics committee approved the analysis of this archival tissue without individual informed consent since the tissue was taken as part of routine care.

\section{Author contributions}

MTR, LBT, MEP, and PE designed the project. All experiments, data acquisition, and data analysis were performed by MTR. SJ and $\mathrm{BM}$ acquired clinical samples. MGJ assisted with RNA extraction. LBT and AL supervised cellular studies. MV and NK undertook RNA sequencing of clinical samples. RNA sequencing of cell culture models was in collaboration with JMD and RX. Bioinformatic data analysis was supervised by MEP and AFV. Analysis of raw sequences from published studies was in collaboration with JR. Immunohistochemistry was supervised by SW and analyzed by SJ. All authors edited and approved the manuscript.

\section{Acknowledgments}

This work was supported by the Rosetrees Trust (M540), Scadding Morriston Davies travel award, MRC Global Challenges Research Fund MR/P023754/1, and NIH grant R33AI102239. MEP is funded by a Sir Henry Dale Fellowship from Wellcome Trust (109377/Z/15/Z) and by the Institute for Life Sciences, University ofSouthampton. NKisfunded by NIH NHLBIgrantsR01HL127349, R01HL141852, U01HL145567, and UH2HL123886. AL was supported by the Wellcome Trust (210662/Z/18/Z) and the Bill and Melinda Gates Foundation (OPP1137006). MGJ acknowledges the support of the Wellcome Trust (100638/Z/12/Z) and the British Lung Foundation (SRG19/10001). We acknowledge support of the NIHR Southampton Biomedical Research Center, the Southampton Histochemistry Research Unit, and Computational Modelling Group, University of Southampton, for assistance with the High Performance Computing Platform. We thank Regina Teo and Jenny Russell for excellent technical support. Graphical abstract was created with BioRender.com 
Address correspondence to: Paul Elkington or Marta Ewa Polak, Clinical and Experimental Sciences, Systems Immunology Group, University of Southampton, Southampton SO16 1YD,
United Kingdom. Phone: 023.8120.5928; Email: p.elkington@ soton.ac.uk (PE); Phone: 023.8120.5727; Email: m.e.polak@ soton.ac.uk (MEP).
1. World Health Organization. Global Tuberculosis Report 2020. World Health Organization; 2020.

2. Opie EL, Aronson JD. Tubercle bacilli in latent tuberculous lesions and in lung tissue without tuberculous lesions. Arch Pathol Lab Me. 1927;4(1):1-21.

3. Cambier CJ, et al. Host evasion and exploitation schemes of Mycobacterium tuberculosis. Cell. 2014;159(7):1497-1509.

4. Russell DG, et al. Tuberculosis: what we don't know can, and does, hurt us. Science. 2010;328(5980):852-856.

5. Valeyre D, et al. Sarcoidosis. Lancet. 2014;383(9923):1155-1167.

6. Calender A, et al. Current insights in genetics of sarcoidosis: functional and clinical impacts. J Clin Med. 2020;9(8):2633.

7. Elkington PT, et al. Tuberculosis immunopathology: the neglected role of extracellular matrix destruction. Sci Transl Med. 2011;3(71):71ps6.

8. Dubos RJ, Dubos J, eds. The White Plague: Tuberculosis, Man, and Society. Rutgers University Press; 1987.

9. Hawn TR, Vandal O. Host-directed therapeutics for tuberculosis: can we harness the host? Microbiol Mol Biol Rev. 2013;77(4):608-627.

10. Zumla A, et al. Host-directed therapies for infectious diseases: current status, recent progress, and future prospects. Lancet Infect Dis. 2016;16(4):e47-e63.

11. Thuong NT, et al. Identification of tuberculosis susceptibility genes with human macrophage gene expression profiles. PLoS Pathog. 2008;4(12):e1000229.

12. Chai Q, et al. Lung gene expression signatures suggest pathogenic links and molecular markers for pulmonary tuberculosis, adenocarcinoma and sarcoidosis. Commun Biol. 2020;3(1):604.

13. Estevez O, et al. An RNA-seq based machine learning approach identifies latent tuberculosis patients with an active tuberculosis profile. Front Immunol. 2020;11:1470.

14. Tezera LB, et al. Dissection of the host-pathogen interaction in human tuberculosis using a bioengineered 3-dimensional model. Elife. 2017;6:e21283.

15. Bielecka MK, et al. A bioengineered threedimensional cell culture platform integrated with microfluidics to address antimicrobial resistance in tuberculosis. mBio. 2017;8(1):e02073-16.

16. Schnute ME, et al. Modulation of cellular S1P levels with a novel, potent and specific inhibitor of sphingosine kinase-1. Biochem J. 2012;444(1):79-88.

17. Kwon YB, et al. Novel synthetic ceramide derivatives increase intracellular calcium levels and promote epidermal keratinocyte differentiation. J Lipid Res. 2007;48(9):1936-1943.

18. Russell DG. Mycobacterium tuberculosis and the intimate discourse of a chronic infection.
Immunol Rev. 2011;240(1):252-268.

19. Lindner B, et al. Phagocytosis assays with different $\mathrm{pH}$-sensitive fluorescent particles and various readouts. Biotechniques. 2020;68(5):245-250.

20. Iannuzzi MC, et al. Medical progress: aarcoidosis. New Engl J Med. 2007;357(21):2153-2165.

21. O'Garra A, Berry MPR. The immune response in tuberculosis. Annu Rev Immunol. 2013;31:475-527.

22. Clayton K, et al. Gene expression signatures in tuberculosis have greater overlap with autoimmune diseases than with infectious diseases. Am J Respir Crit Care Med. 2017;196(5):655-656.

23. Tezera LB, et al. Reconsidering the optimal immune response to Mycobacterium tuberculosis. Am J Respir Crit Care Med. 2020;201(4):407-413.

24. Ong CWM, Friedland JS. Tuberculosis, pulmonary cavitation, and matrix metalloproteinases. Am J Respir Crit Care Med. 2014;190(1):9-18.

25. Elkington P, Friedland JS. MMP-1 drives immunopathology in human tuberculosis and transgenic mice. J Clin Invest. 2011;121(5):1827-1833.

26. Balbin $\mathrm{M}$, et al. Identification and enzymatic characterization of two diverging murine counterparts of human interstitial collagenase (MMP-1) expressed at sites of embryo implantation. J Biol Chem. 2001;276(13):10253-10262.

27. Al Shammari B, et al. The extracellular matrix regulates granuloma necrosis in tuberculosis. J Infect Dis. 2015;212(3):463-473.

28. Ordonez AA, et al. Matrix metalloproteinase inhibition in a murine model of cavitary tuberculosis paradoxically worsens pathology. J Infect Dis. 2019;219(4):633-636.

29. Urbanowski ME, et al. Repetitive aerosol exposure promotes cavitary tuberculosis and enables screening for targeted inhibitors of extensive lung destruction. J Infect Dis. 2018;218(1):53-63.

30. Xu Y, et al. Matrix metalloproteinase inhibitors enhance the efficacy of frontline drugs against Mycobacterium tuberculosis. PLoS Pathog. 2018;14(4):e1006974.

31. Hao Miow Q, et al. Doxycycline host-directed therapy in human pulmonary tuberculosis. J Clin Invest. 2021:141895.

32. Bellamy R, et al. Variations in the NRAMP1 gene and susceptibility to tuberculosis in West Africans. N Engl J Med. 1998;338(10):640-644.

33. Archer NS, et al. Genetic variants of SLC11A1 are associated with both autoimmune and infectious diseases: systematic review and meta-analysis. Genes Immun . 2015;16(4):275-283.

34. Davis JM, Ramakrishnan L. The role of the granuloma in expansion and dissemination of early tuberculous infection. Cell. 2009;136(1):37-49.

35. Rual JF, et al. Towards a proteome-scale map of the human protein-protein interaction network. Nature. 2005;437(7062):1173-1178.

36. Russell DG, et al. Foamy macrophages and the progression of the human tuberculosis granuloma. Nat Immunol. 2009;10(9):943-948.

37. Hansson GK, Libby P. The immune response in atherosclerosis: a double-edged sword. Nat Rev Immunol. 2006;6(7):508-519.

38. Li D, et al. LOX-1 inhibition in myocardial ischemia-reperfusion injury: modulation of MMP-1 and inflammation. Am J Physiol Heart Circ Physiol. 2002;283(5):H1795-H1801.

39. Bekkering S, et al. Oxidized low-density lipoprotein induces long-term proinflammatory cytokine production and foam cell formation via epigenetic reprogramming of monocytes. Arterioscler Thromb Vasc Biol. 2014;34(8):1731-1738.

40. Nakagawa TY, Rudensky AY. The role of lysosomal proteinases in MHC class II-mediated antigen processing and presentation. Immunol Rev. 1999;172:121-129.

41. Scriba TJ, et al. Sequential inflammatory processes define human progression from $\mathrm{M}$. tuberculosis infection to tuberculosis disease. PLoS Pathog. 2017;13(11):e1006687.

42. Berry MP, et al. An interferon-inducible neutrophil-driven blood transcriptional signature in human tuberculosis. Nature. 2010;466(7309):973-977.

43. Kim MJ, et al. Caseation of human tuberculosis granulomas correlates with elevated host lipid metabolism. EMBO Mol Med. 2010;2(7):258-274.

44. Tekin H, et al. Effects of 3D culturing conditions on the transcriptomic profile of stem-cell-derived neurons. Nat Biomed Eng. 2018;2(7):540-554.

45. Schwartz MA, Chen CS. Cell biology. Deconstructing dimensionality. Science. 2013;339(6118):402-404.

46. Yuki K, et al. Organoid models of tumor immunology. Trends Immunol. 2020;41(8):652-664.

47. Tezera LB, et al. Anti-PD-1 immunotherapy leads to tuberculosis reactivation via dysregulation of TNF- $\alpha$. Elife. 2020;9:e52668.

48. Ogongo P, et al. Tissue resident-like CD4+ T cells secreting IL-17 control Mycobacteria tuberculosis in the human lung. J Clin Invest. 2021;131(10):e142014.

49. Spiegel S, Milstien S. Sphingosine-1-phosphate: an enigmatic signalling lipid. Nat Rev Mol Cell Biol. 2003;4(5):397-407.

50. Maceyka M, et al. Sphingosine-1-phosphate signaling and its role in disease. Trends Cell Biol. 2012;22(1):50-60.

51. Vergne I, et al. Mechanism of phagolysosome biogenesis block by viable Mycobacterium tuberculosis. Proc Natl Acad Sci U S A. 2005;102(11):4033-4038.

52. Anes E, et al. Selected lipids activate phagosome actin assembly and maturation resulting in killing of pathogenic mycobacteria. Nat Cell Biol. 2003;5(9):793-802.

53. Thompson CR, et al. Sphingosine kinase 1 (SK1) is recruited to nascent phagosomes in human macrophages: inhibition of SK1 translocation 
by Mycobacterium tuberculosis. J Immunol. 2005;174(6):3551-3561.

54. DiNardo AR, et al. Tuberculosis endotypes to guide stratified host-directed therapy. Cell. 2021;2(3):217-232.

55 . Andreu N, et al. Optimisation of bioluminescent reporters for use with mycobacteria. PLoS One. 2010;5(5):e10777.

56 . Workman VL, Jayasinghe SN. Controlled generation of microspheres incorporating extracellular matrix fibrils for three-dimensional cell culture. Adv Funct Mater. 2014;24(8):2648-2657.

57. Bray NL, et al. Near-optimal probabilistic RNA-seq quantification. Nat Biotechnol. 2016;34(5):525-527.
58. Soneson C, et al. Differential analyses for RNA-seq: transcript-level estimates improve gene-level inferences. F100ORes. 2015;4:1521.

59. Rainer J, et al. ensembldb: an R package to create and use Ensembl-based annotation resources. Bioinformatics. 2019;35(17):3151-3153.

60. Pimentel H, et al. Differential analysis of RNAseq incorporating quantification uncertainty. Nat Methods. 2017;14(7):687-690.

61. Ritchie ME, et al. limma powers differential expression analyses for RNA-sequencing and microarray studies. Nucleic Acids Res. 2015;43(7):e47.

62. Theocharidis A, et al. Network visualization and analysis of gene expression data using BioLayout
Express(3D). Nat Protoc. 2009;4(10):1535-1550.

63. Subramanian A, et al. Gene set enrichment analysis: a knowledge-based approach for interpreting genome-wide expression profiles. Proc Natl Acad Sci U S A. 2005;102(43):15545-15550.

64. Alhamdoosh M, et al. Combining multiple tools outperforms individual methods in gene set enrichment analyses. Bioinformatics. 2017;33(3):414-424.

65. Yu G, He QY. ReactomePA: an R/Bioconductor package for reactome pathway analysis and visualization. Mol Biosyst. 2016;12(2):477-479.

66. Chen J, et al. ToppGene Suite for gene list enrichment analysis and candidate gene prioritization. Nucleic Acids Res. 2009;37(Suppl_2):W305-W311. 